\title{
Review \\ The effect of pressotherapy on performance and recovery in the management of delayed onset muscle soreness: A Systematic Review and Meta-Analysis
}

\author{
Paweł Wiśniowski ${ }^{1}$, Maciej Cieśliński ${ }^{1}$, Martyna Jarocka ${ }^{1}$, Przemysław Seweryn Kasiak ${ }^{2}$, Bartłomiej Makaruk ${ }^{1}$, \\ Wojciech Pawliczek ${ }^{1}$ and Szczepan Wiecha ${ }^{1, *}$ \\ 1 Department of Physical Education and Health in Biala Podlaska, Faculty in Biala Podlaska, Jozef Pilsudski \\ University of Physical Education in Warsaw, Biala Podlaska, 21-500 Biala Podlaska; Poland; \\ szczepan.wiecha@awf.edu.pl \\ $23^{\text {Rd }}$ Department of Internal Medicine and Cardiology Medical University of Warsaw, 02-091, Warsaw, \\ Poland \\ * Correspondence: szczepan.wiecha@awf.edu.pl; Tel.: +48-833-428-823
}

\begin{abstract}
Background: It has been demonstrated that pressotherapy used post-exercise (Po-E) can influence training performance, recovery, and physiological properties. This study examined the effectiveness of pressotherapy on these parameters. Methods: The systematic review and metaanalysis were performed according to PRISMA guidelines. A literature search of MEDLINE, PubMed, EBSCO, Web of Science, SPORTDiscus and ClinicalTrials has been done up to March 2021. Inclusion criteria were: randomized control trials (RCTs) or cross-over studies, mean participant age between 18-65 yrs., $\geq 1$ exercise mechanical pressotherapy intervention. The risk of bias was assessed by the Cochrane risk-of-bias tool for RCT (RoB 2.0). Results: 12 studies comprised of 322 participants have been selected. The mean sample size was $n=25$. Pressotherapy significantly reduce muscle soreness(Standard Mean Difference;SMD = -0.33; $\mathrm{CI}=-0.49,-0.18 ; \mathrm{p}<0.0001 ; \mathrm{I}^{2}=7 \%$ ). Pressotherapy did not significantly affect jump height $(\mathrm{SMD}=-0.04 ; \mathrm{CI}=-0.36,-0.29 ; \mathrm{p}=0.82)$. Pressotherapy did not significantly affect creatine kinase level 24-96h after DOMS induction (SMD $=0.41 ; \mathrm{CI}=-0.07$, $\left.0.89 ; \mathrm{p}=0.09 ; \mathrm{I}^{2}=63 \%\right)$. Conclusions: Only moderate benefits of using pressotherapy as a recovery intervention have been observed. Results varied between the type of exercise and used protocol. Pressotherapy should only be applied as an additional component of a more comprehensive recovery strategy. Study PROSPERO registration number- CRD42020189382.
\end{abstract}

Keywords: pressotherapy; compression; regeneration; DOMS

\section{Introduction}

Physical activity, especially at the competitive level, causes a lot of negative changes in the human body [2][3]. Inflammation occurs as a result of damage to muscle cells [4] from which creatine kinase (CK), lactate dehydrogenase, and metabolites are released [2][3]. In such cases, we observe decreased efficiency, faster muscle fatigue, a decrease in the range of motion (ROM), and the appearance of pain in places where they are overloaded [5][6]. This phenomenon is exacerbated especially with eccentric exercises (ECC) [7], in which intense exercise may cause Delayed Onset Muscle Soreness (DOMS) [8].

To increase exercise capacity as well as reduce the risk of injury, the key element is the use of training measures related to biological recovery to reduce metabolites to minimum values and to ensure the right amount of energy substrates, including ATP and phosphocreatine [9]. 
The most commonly used methods of biological recovery include treatments in the field of physical therapy (cold therapy, heat therapy, electrotherapy, compression therapy), manual therapy, massage (myofascial release and self-myofascial release), and pharmacology [10][11]. Of the above methods, in recent years much attention has been paid to compression therapy [12]), in which the most frequent mention is External Pneumatic Compression (EPC) [13] as well as Intermittent Pneumatic Compression (IPC) [13].

Among the studies that used EPCs, a positive effect was found to increase flexibility and reduce muscle soreness (MS) [14][15], as well as reducing lymphoedema [16] and reduction of lactate [17]. The research conducted by Martin et al. [2015] showed that EPC did not statistically significantly affect the reduction of lactate after the 30-second Wingate test compared to the control group. Similar relationships were found by Haun et al. [2017], in which they did not notice a statistical difference in muscle strength between the control group and the experimental group after resistance training in the form of back squats.

Using IPC has been reported to be effective in regeneration with short-term ECC efforts, reduction of fatigue [18], reduction of edema [19], improvement of local blood supply [20] and improve the ROM [13]. In subsequent studies, IPC was more effective at reducing high lactate levels than passive rest after exercise [21], and also statistically significantly reduced soft tissue stiffness after ECC training [19] and slightly reduced delayed post-exercise (Po-E) pain after short-term intense exercise [22].

Other studies have shown mitigating the effects of reducing muscle strength immediately after training [18] and improving the speed of a 400-meter run [13].

This systematic review and meta-analysis aimed to examine the effectiveness of the above forms of compression therapy to reduce DOMS. The primary endpoint is to assess pressotherapy the changes in MS and sports performance. The secondary endpoint is to establish the specific benefits on the selected outcomes of muscle functional capacities (e. g. strength, power), muscle damage markers (e. g. serum CK levels), joint ROM and pain sensation

\section{Materials and Methods}

The present review and meta-analysis were reported according to the Preferred Reporting Items for Systematic Reviews and Meta-Analyses (PRISMA) and follow the recommendations of the Cochrane Handbook for Systematic Reviews of Interventions [23]. The PRISMA 2020 statement: an updated guideline for reporting systematic reviews. Systematic Reviews [1].

\subsection{Search Strategy and Screening Procedures}

Searches were carried out on the following electronic databases: MEDLINE (PubMed and EBSCO), Web of Science, SPORT Discus, we didn't have any limits and we searched all articles to March 2021 for studies aimed at determining the effect of pressotherapy on the magnitude and time course of Po-E muscle soreness and sports performance and recovery following exercise-induced muscle damage. We also searched current information about registers and reports in ClinicalTrials.gov. We head the same keywords as in databases. There were no associated publications, reports, or registers.

The search algorithm was conducted using PICO's strategy [23] (type of studies, participants, interventions, comparators, and outcome assessment) and combined Medical Subject Headings, free-terms and matching synonyms of the following related words: (1) population: healthy adults, "middle-aged", "young adults"; (2) intervention: external assisted mechanical therapy, „external counterpulsation", "lymphatic drainage", "pressotherapy", "intermittent pneumatic compression", "pneumatic compression", "pneumatic therapy", "intermittent compression", "compression therapy", "compression massage", "pneumatic massage")"; (3) outcome: "Soreness", "DOMS", "inflammation", "muscle fatigue", "recovery", "Delayed Onset Muscle Soreness", "EIMD", 
"hyperalgesia", "allodynia", "myalgia"; and (4) comparator: control conditions; RCT's studies and cross-over. In addition, we searched the citations included in the identified publications deemed eligible for our study.

\subsection{Inclusion Criteria}

Those studies in which the title and abstract were related to the aim of the present review were included for full-text request. We included studies that (1) were conducted as randomized control trials (RCT) and cross-over designs; (2) included a mean participant age between 18 and 65 y.o. (3) Healthy adults with exercise-induced muscle damage regardless of their level of sports activity and performance (4) were based on at least one exercise intervention described as "External assisted mechanical therapy" (machines).

\subsection{Exclusion Criteria}

Studies were excluded if (1) outcome measurements were not reported as DOMS max values, or (2) they were not written in English. A third reviewer (SW) resolved cases of initial reviewer disagreement. Nonrandomized experiments, observational studies, secondary studies (any types of evidence syntheses), and opinion pieces (e. g. narrative reviews, editorials) were excluded too.

\subsection{Selection process, data collection, data extraction, and management}

Two initial reviewers (MJ and MC) independently examined the titles and abstracts of retrieved articles to identify suitable studies and extracted the following information from the included studies: First author's name and year of publication; study design; characteristics of the participants included; mean age; sample size and percentage of female subjects; weekly frequency, period and modality of External assisted mechanical therapy intervention; the reported measurement of Muscle functional capacities (e. $g$. strength, power), Muscle damage markers (e.g. serum CK levels), Joint ROM and pain sensation. A third reviewer (SW) resolved cases of author disagreement.

\subsection{Risk of Bias Assessment}

The risk of bias of RCTs was assessed using the Cochrane risk-of-bias tool for randomized trials (RoB 2.0) [24], in which five domains were evaluated: Randomization process, deviations from intended interventions, missing outcome data, measurement of the outcome, and selection of the reported result. Each domain was assessed for risk of bias. Studies were graded as (1) "low risk of bias" when a low risk of bias was determined for all domains; (2) "some concerns" if at least one domain was assessed as raising some concerns but not to be at high risk of bias for any single domain; or (3) "high risk of bias" when a high risk of bias was reached for at least one domain or the studied judgment included some concerns in multiple domains [1]. For pre-post studies and non-RCTs we used the Quality Assessment Tool for Quantitative Studies [24], in which seven domains were evaluated: Selection bias, study design, confounders, blinding, data collection methods, withdrawals, and dropouts. Each domain was considered strong, moderate, or weak. Studies were classified as "low risk of bias" if they presented no weak ratings; "moderate risk of bias" when there was at least one weak rating; or "high risk of bias" if there were two or more weak ratings [24]. The risk of bias was independently assessed by two reviewers (MJ and PW). A third reviewer (SW) was consulted in case of disagreement. 


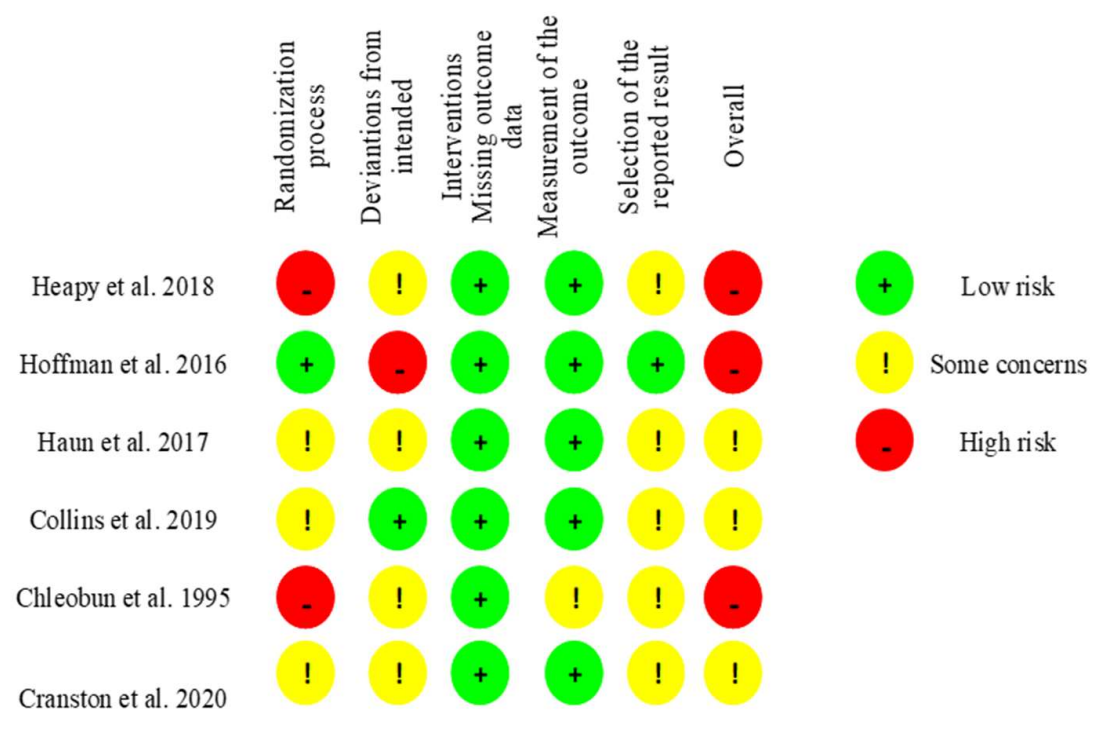

Figure 1. Risk of bias 2 tool. Assessment for individual randomized, parallel-group trials.

\begin{tabular}{|c|c|c|c|c|c|c|}
\hline 晜 & 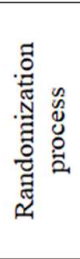 & 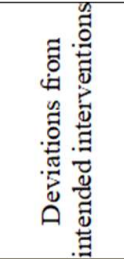 & 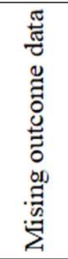 & 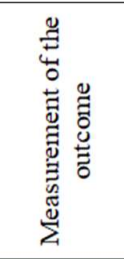 & 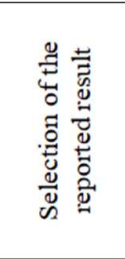 & 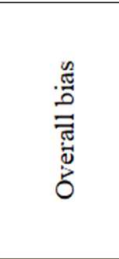 \\
\hline Cochrane et al. 2013 & High & $\begin{array}{c}\text { Some } \\
\text { concerns }\end{array}$ & Low & Low & $\begin{array}{c}\text { Some } \\
\text { concerns }\end{array}$ & $\begin{array}{c}\text { Some } \\
\text { concerns }\end{array}$ \\
\hline Draper et al. 2020 & High & $\begin{array}{c}\text { Some } \\
\text { concerns }\end{array}$ & Low & Low & $\begin{array}{c}\text { Some } \\
\text { concerns }\end{array}$ & High \\
\hline Northey et al. 2016 & High & $\begin{array}{c}\text { Some } \\
\text { concerns }\end{array}$ & Low & $\begin{array}{c}\text { Some } \\
\text { concerns }\end{array}$ & $\begin{array}{c}\text { Some } \\
\text { concerns }\end{array}$ & $\begin{array}{c}\text { Some } \\
\text { concerns }\end{array}$ \\
\hline Velanzuela et al. 2018 & High & $\begin{array}{c}\text { Some } \\
\text { concerns }\end{array}$ & Low & Low & $\begin{array}{c}\text { Some } \\
\text { concerns }\end{array}$ & High \\
\hline Oliver et al. 2021 & High & High & Low & Low & $\begin{array}{c}\text { Some } \\
\text { concerns }\end{array}$ & High \\
\hline
\end{tabular}

Figure 2. Risk of bias 2 tool. Individually randomized, cross-over trials.

2.6. Outcome measures.

Objective results of interest for meta-analyses from included baseline to last available follow-up. Data were typically collected immediately and $24 \mathrm{~h}, 48 \mathrm{~h}, 72 \mathrm{~h}$, up to $96 \mathrm{~h}$ after the intervention.

\subsection{Primary outcomes}

The primary endpoint is to assess the effect changes in MS and sports performance.

\subsection{Secondary outcomes}

The secondary endpoint is to muscle functional capacities (e. g. strength, power), muscle damage markers (e. g. serum CK levels), and joint ROM and pain sensation.

\section{Results}




\subsection{Results of the Search}

A total of 693 articles related to the topic were retrieved through a comprehensive database and other sources search, of which, 169 articles were duplicates. After removing all ineligible articles a total of 12 RCTs were included in the analysis. The detailed screening process is shown in (Figure 3).

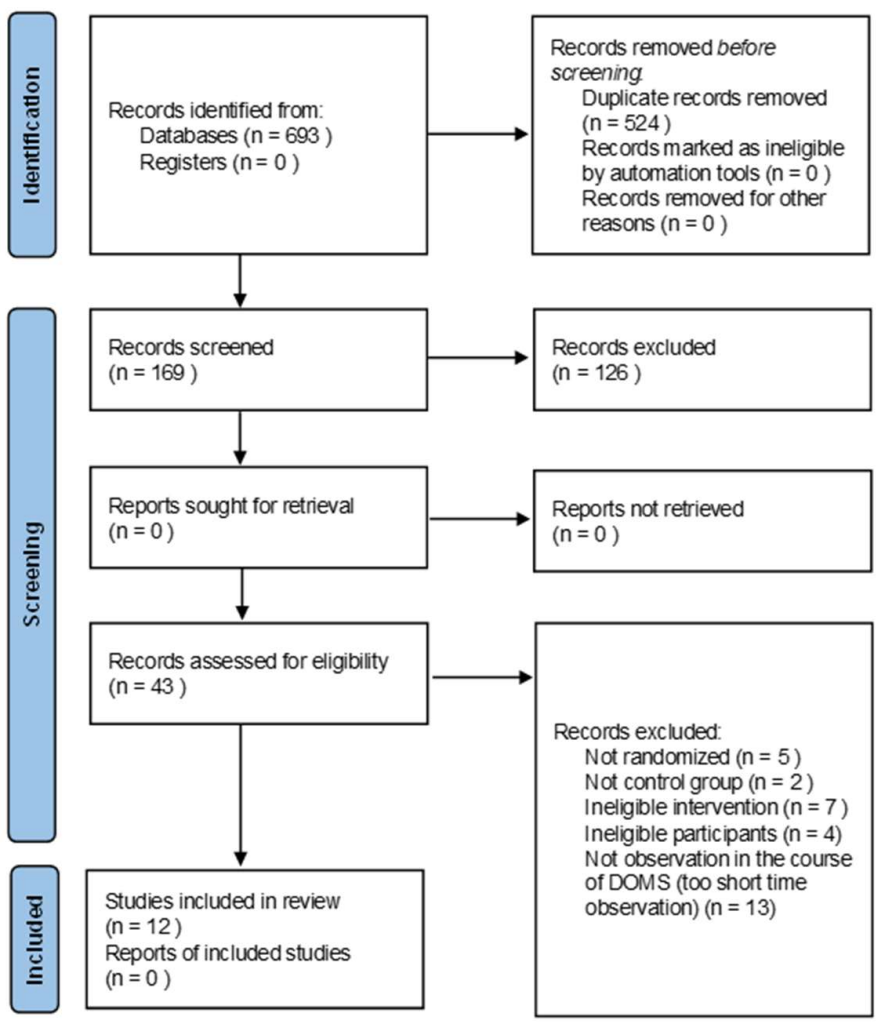

Figure 3. PRISMA flow diagram of included/excluded studies.

\subsection{Details of the intervention groups in the included studies.}

Characteristics of the included studies are summarized in Table 1.

Table 1. The key characteristic of selected studies $(n=12)$.

There were 5 randomized controlled trials [18][25][26][27][28] and 7 randomized crossover trials [29][13][12][30][31][19][32]. Overally, studies included patients from five countries: USA $(n=5)$, New Zealand $(n=3)$, Ireland $(n=1)$, Australia $(n=2)$, and Spain (n $=1)$.

The total study population of all selected articles comprised of 322 healthy volunteers with an unequal distribution of sex ( $\left.\mathrm{n}_{\text {male }}=274 ; \mathrm{n}_{\mathrm{female}}=48\right)$. Throughout all the studies, mean sample size ranged from 10 to 72 volunteers).

The average sample size of the pressotherapy group was 14.33 and the control group 13.25. The mean age of the study population was $28.1 \mathrm{yrs}$. In two studies the mean age was above 40 yrs. [2,3].

Two studies involved well-trained volunteers [12][28]. Three studies included runners [29][13][25]. One study included strength-trained males [26]. Two studies included physically active volunteers [30][18] and athletes [31][32], another two studies chose healthy participants [19][27]. Detailed information about the training status is presented in the Table 1.

\subsection{Characteristics of the Exercise Protocols, Therapy \& Outcomes}


To induce muscle damage exercise protocols encompassed run and other activities. Five used run [29][13][12][31][25]. One of these types of exercise was sprint [31], another one middle-6km [12] and three while the remaining five were long-distance run 62,7 [29]; 87,4 [29]; 102,8 [29]; $2 \times 20$ mile [25]; $161 \mathrm{~km}$ [13]. Two studies used back squats, 10 sets x10 rep [26], and 10 sets of 5 repetition [30]. Another way to induction DOMS intervention was ECC exercise on Biodex system [18], eccentric exercise performed with weight [19], plyometric exercise bout [27], countermovement jump (CMJ) [31], and wheelchair court sprints [28]. One study used specific training: Reverse grip battle rope waves, Farmers carry, Chin-ups, Bar hangs, Handgrip crushers [32]. Table 1 gives a detailed overview of the conducted exercise protocols.

Table 1. The key characteristic of selected studies $(n=12)$

\begin{tabular}{|c|c|c|c|c|c|c|c|c|c|}
\hline $\begin{array}{c}\text { Author/co } \mathrm{P} \\
\text { untry }\end{array}$ & $\begin{array}{l}\text { Design / } \\
\text { Publicati } \\
\text { on } \\
\text { year }\end{array}$ & $\begin{array}{l}\text { Participant } \\
\text { cohort } \\
\text { (training } \\
\text { status, sex, } \\
\text { age) }\end{array}$ & $\begin{array}{l}\text { Sample } \\
\text { Size } \\
\text { (n) }\end{array}$ & $\begin{array}{l}\text { Experimen } \\
\text { tal vs. } \\
\text { control } \\
\text { condition }\end{array}$ & $\begin{array}{l}\text { DOMS induction } \\
\text { intervention }\end{array}$ & $\begin{array}{c}\text { Outcome } \\
\text { variables and } \\
\text { time of } \\
\text { measurement } \\
\text { post-exercise } \\
\text { (hrs) }\end{array}$ & $\begin{array}{l}\text { Main effects }[* p<0,05: \text { pre-post } \\
\qquad(x \text { time })]\end{array}$ & $\begin{array}{l}\text { Total } \\
\text { exposition } \\
\text { time }\end{array}$ & Therapy parameters \\
\hline $\begin{array}{l}\text { Hoffman } \\
\text { et. al. / USA }\end{array}$ & $\begin{array}{l}\text { RCT / } \\
2016\end{array}$ & $\begin{array}{l}\text { participants in } \\
\text { the } 2015 \text { 161- } \\
\text { km Western } \\
\text { States } \\
\text { Endurance } \\
\text { Run, men } \\
\text { (IPC: } 43 \pm 8 \\
\text { yrs., Massage: } \\
46 \pm 10 \text { yrs., } \\
\text { con.: } 45 \pm 9 \text { yrs) }\end{array}$ & $\begin{array}{c}n=72 \\
\\
n=24 \\
\text { exp. (IPC) } \\
n=25 \\
\text { exp. } \\
\text { (Massage( } \\
\text { ) } \\
n=23 \\
\text { con. }\end{array}$ & $\begin{array}{l}\text { 45min post } \\
\text { exercises } \\
\text { IPC (20 } \\
\text { min), } \\
45 \mathrm{~min} \\
\text { post- } \\
\text { exercise } \\
\text { Massage } \\
\text { (20min) vs. } \\
\text { Placebo } \\
\text { therapy } \\
\text { (20 min) }\end{array}$ & $\begin{array}{c}161 \mathrm{~km} \\
\text { ultramarathon } \\
\text { race }\end{array}$ & $\begin{array}{l}\text { 400-m run } \\
\text { times, Muscle } \\
\text { Pain and } \\
\text { Soreness, } \\
\text { Overall Fatigue } \\
\text { (prerace, } \\
\text { postrace, } \\
\text { posttreatment, } \\
\text { 24h-168h post- } \\
\text { race day) }\end{array}$ & $\begin{array}{c}\text { 400-m run time's (pre } \leftrightarrow, \text { post } \\
72 \mathrm{~h} \uparrow, 120 \mathrm{~h} \downarrow \text { ) } \\
\text { Lower-Body Muscle Pain and } \\
\text { Soreness (pre } \leftrightarrow, \text { postrace } \uparrow^{*}, \\
\text { posttreatment }{ }^{*} \#, \text { post } 24 \mathrm{~h}- \\
96 \uparrow^{*}, \text { post-120h-168 } \mathrm{h} \uparrow \\
\text { Time and interaction effect* (no } \\
\text { group effect) } \\
\text { Muscular Fatigue (pre } \leftrightarrow, \\
\text { postrace } \uparrow, \text { post-treatment } \uparrow^{*} \#, \\
\text { postrace } 24 \mathrm{~h}-168 \mathrm{~h} \uparrow \text { ) } \\
\text { Time and interaction effect* (no } \\
\text { group effect) }\end{array}$ & $\begin{array}{l}20 \text { min ISPC } \\
20 \text { min } \\
\text { Massage } 20 \\
\text { min Con. }\end{array}$ & $\begin{array}{l}\text { ISPC - } 80 \mathrm{mmHg} \\
\text { Massage - (the } 30 \mathrm{~s} \text { - } \\
\text { calf and hamstring, } \\
1 \text { min - quadriceps), } \\
\text { compression ( } 2 \mathrm{~min} \\
\text { - calf and } \\
\text { quadriceps, } 3 \mathrm{~min} \\
\text { hamstring), } \\
\text { tapotement (30s leg } \\
\text { and quadriceps) }\end{array}$ \\
\hline $\begin{array}{l}\text { Haun et. al } \\
\text { / USA }\end{array}$ & $\begin{array}{l}\text { RCT / } \\
2017\end{array}$ & $\begin{array}{l}\text { endurance- } \\
\text { trained male, } \\
\text { participating in } \\
\geq 72 \mathrm{~h} \text { per week } \\
\text { of endurance } \\
\text { exercise for at } \\
\text { least } 3 \\
\text { months. } \\
\text { (EPC: } 21 \pm 0.4 \\
\text { yrs, } \\
\text { con: } 21.1 \pm 0.6 \\
\text { yrs) }\end{array}$ & $\begin{array}{c}\mathrm{n}=18 \\
\mathrm{n}=9 \\
\exp . \\
(\mathrm{EPC}) \\
\mathrm{n}=9 \text { con. } \\
\end{array}$ & $\begin{array}{c}24 \mathrm{~h}, 48 \mathrm{~h}, \\
72 \mathrm{~h} \text { post } \\
\text { exercises } \\
\text { EPC (1h) } \\
\text { vs. Placebo } \\
\text { therapy } \\
(1 \mathrm{~h}) \\
96 \mathrm{~h}, 120 \mathrm{~h} \\
\text { treatments } \\
\text { only EPC } \\
\text { (1h) vs. } \\
\text { placebo } \\
\text { therapy } \\
\text { (1h) }\end{array}$ & $\begin{array}{c}6 \mathrm{~km} \text { run on the } \\
\text { treadmill at an } \\
\text { incline of } 1 \% \text { (pre } \\
\text { and } 16 \mathrm{~h} \text { ) }\end{array}$ & $\begin{array}{c}\text { CK, Muscle Pain, } \\
\text { and Soreness } \\
\text { (pre-exercises, } \\
72 \mathrm{~h} \text { to } 168 \mathrm{~h} \text { ), } \\
\text { Flexibility (pre- } \\
\text { exercises, } 72 \mathrm{~h} \\
\text { to } 168 \mathrm{~h}), 6-\mathrm{km} \\
\text { run times (pre- } \\
\text { exercises, } 168 \mathrm{~h} \text { ) }\end{array}$ & $\begin{array}{c}\text { CK (pre, } 72 \mathrm{~h} \uparrow, 96 \mathrm{~h} \uparrow *, 120 \mathrm{~h} \uparrow, \\
144 \mathrm{~h} \uparrow, 168 \mathrm{~h} \leftrightarrow \text { ) } \\
\text { Time effect* (No group or group x } \\
\text { interaction effect) } \\
\text { Muscle Soreness (pre, } 72 \mathrm{~h} \downarrow *, \\
96 \mathrm{~h} \downarrow, 120 \mathrm{~h} \downarrow *, 144 \mathrm{~h} \downarrow, 168 \mathrm{~h} \leftrightarrow \text { ) } \\
\text { Time effect* (No group or time - } \\
\text { group effect) } \\
\text { Flexibility (pre, } 72 \mathrm{~h} \uparrow, 96 \mathrm{~h} \leftrightarrow, \\
120 \mathrm{~h} \leftrightarrow, 144 \mathrm{~h} \leftrightarrow, 168 \mathrm{~h} \downarrow) \\
6 \mathrm{~km} \text { run time (pre, } 168 \mathrm{~h} \downarrow)\end{array}$ & $\begin{array}{l}300 \min \text { EPC } \\
300 \min \text { EPC } \\
\text { Con }\end{array}$ & $\begin{array}{cc}C & \text { EPC }-70 \mathrm{mmHg} \\
C & \text { (inflation - 30s / } \\
& \text { deflation - 30s) }\end{array}$ \\
\hline $\begin{array}{l}\text { Cochrane } \\
\text { et. al / NZ }\end{array}$ & $\begin{array}{l}\mathrm{RCO} / \\
2013\end{array}$ & $\begin{array}{l}10 \text { healthy } \\
\text { males, } \\
\text { involved in } \\
\text { physical } \\
\text { activity ( } 21.0 \pm \\
1.7 \text { yrs) }\end{array}$ & $\begin{array}{c}n=10 \\
n=10 \\
\text { exp. (IPC) } \\
n=10 \\
\text { con. }\end{array}$ & $\begin{array}{l}\text { immediate } \\
\text { ly post } \\
\text { exercises, } \\
24 \mathrm{~h} \text { post- } \\
\text { exercise, } \\
48 \mathrm{~h} \text { post } \\
\text { IPC ( } 30 \\
\text { min) vs. } \\
\text { Placebo } \\
\text { therapy } \\
\text { (30 min) }\end{array}$ & $\begin{array}{l}3 \text { sets } \times 100 \text { rep. } \\
\text { strenuous bout of } \\
\text { eccentric exercise } \\
\text { on BIODEX }\end{array}$ & $\begin{array}{c}\text { CK, VJ, Muscle } \\
\text { Dynamometry } \\
\text { ISO } 75^{\circ} \\
\text { - CON } 30^{\circ} / \mathrm{sec} ; \\
180^{\circ} / \mathrm{sec} \\
\text { - ECC } 30^{\circ} / \mathrm{sec} ; \\
180^{\circ} / \mathrm{sec} \text { (Pre, } \\
24 \mathrm{~h}, 48 \mathrm{~h} \text {, post } \\
72 \mathrm{~h})\end{array}$ & 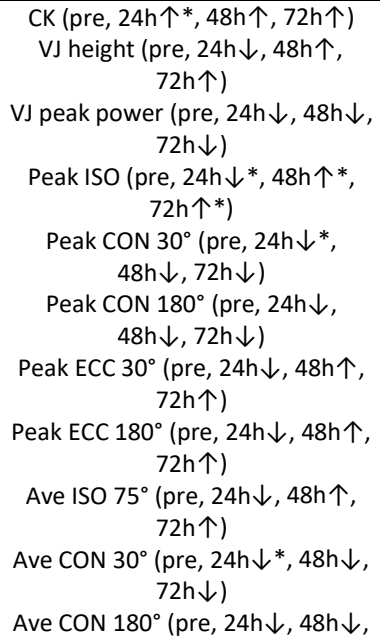 & $\begin{array}{l}\text { 90min IPC } \\
90 \mathrm{~min} \text { Con }\end{array}$ & $\begin{array}{l}\text { IPC - cell } 1 \text { (distal) - } \\
70 \mathrm{mmHg} \text {, cells } 2-4 \\
80 \mathrm{mmHg} \text {, cell } 5 \\
\text { (proximal) } 60 \mathrm{mmHg} \\
\text { / deflation }-30 \mathrm{~s} .\end{array}$ \\
\hline
\end{tabular}




\begin{tabular}{|c|c|c|c|c|c|c|c|c|c|}
\hline & & & & & & & $\begin{array}{c}72 \mathrm{~h} \downarrow) \\
\text { Ave ECC } 30^{\circ} \text { (pre, 24h } \downarrow, 48 \mathrm{~h} \uparrow, \\
72 \mathrm{~h} \uparrow) \\
\text { Ave ECC } 180^{\circ} \text { (pre, } 24 \mathrm{~h} \downarrow, 48 \mathrm{~h} \uparrow, \\
72 \mathrm{~h} \uparrow) \\
\end{array}$ & & \\
\hline $\begin{array}{l}\text { Collins et. } \\
\text { al / IE }\end{array}$ & $\begin{array}{l}\text { RCT / } \\
2019\end{array}$ & $\begin{array}{l}21 \text { male team } \\
\text { sport athletes } \\
(21.6 \pm 3.4 \text { yrs })\end{array}$ & $\begin{array}{c}n=21 \\
n=11 \\
\text { exp. } \\
n=10 \\
\text { con. }\end{array}$ & $\begin{array}{c}\text { pre, post, } \\
24 \mathrm{~h} \text { post } \\
\text { exercises } \\
\text { ECP }(20 \\
\text { min) vs. } \\
\text { Placebo } \\
\text { therapy } \\
(20 \mathrm{~min})\end{array}$ & $\begin{array}{l}\text { Max CMJ, } 2 \times 20 \\
\text { sprint, and second } \\
\text { max CMJ }\end{array}$ & $\begin{array}{c}\text { CK, C, T, IgA, } \\
\text { sAA, VAS, CMJ } \\
\text { height (Pre, } \\
\text { post, } 24 \mathrm{~h} \text { post) }\end{array}$ & $\begin{array}{c}\text { CK (pre, post } \uparrow^{*}, 24 \mathrm{~h} \uparrow * \text { ) Main } \\
\text { effect for time* Cortisol (pre, } \\
\text { post } \uparrow, 24 \mathrm{~h} \downarrow \text { ) } \\
\text { Testosterone (pre, post }{ }^{*}, \\
24 \mathrm{~h} \downarrow^{*} \text { ) Main effect for time } \\
\text { Alpha-Amylase (pre, post } \uparrow^{*} \#, \\
24 \mathrm{~h} \uparrow * \#) \\
\text { Main effect for time, and group } \\
\text { Immunoglobulin - A (pre, } \\
\text { post }, 24 \mathrm{~h} \downarrow \text { ) } \\
\text { VAS (pre, post } \uparrow, 24 \mathrm{~h} \uparrow * \text { ) Main } \\
\text { effect for time } \\
\left.\text { CMJ (pre, post } \downarrow^{*} \#, 24 \mathrm{~h} \uparrow * \#\right)\end{array}$ & $\begin{array}{l}60 \mathrm{~min} \text { ECP } \\
60 \mathrm{~min} \text { Con }\end{array}$ & $\begin{array}{c}\text { ECP }-235.3 \pm \\
26.9 \mathrm{mmHg}\end{array}$ \\
\hline $\begin{array}{l}\text { Draper et. } \\
\text { al / USA }\end{array}$ & $\begin{array}{l}\text { RCO / } \\
2020\end{array}$ & $\begin{array}{c}10 \text { runners, } \\
\text { endurance- } \\
\text { trained males } \\
(38.7 \pm 11.2 \\
\text { yrs })\end{array}$ & $\begin{array}{l}n=10 \\
n=10 \\
\text { exp. } \\
n=10 \\
\text { con. }\end{array}$ & $\begin{array}{c}\text { 1h, } 24 \mathrm{~h}, \\
48 \mathrm{~h}, 72 \mathrm{~h}, \\
96 \mathrm{~h}, 120 \mathrm{~h} \\
\text { post IPC } \\
(1 \mathrm{~h}) \mathrm{vs} . \\
1 \mathrm{~h}, 24 \mathrm{~h}, \\
48 \mathrm{~h}, 72 \mathrm{~h}, \mathrm{~s} \\
96 \mathrm{~h}, 120 \mathrm{~h} \\
\text { post } \\
\text { Placebo } \\
\text { therapy } \\
\text { (1h) }\end{array}$ & $\begin{array}{c}2 \times 20 \text { mile runs at } \\
70 \% \text { VO2 max } \\
\text { separated by } 3 \text { or } 4 \\
\text { weeks }\end{array}$ & $\begin{array}{l}\text { CRP, VAS (pre, } \\
\text { post, and } 24 \mathrm{~h}, \\
448 \mathrm{~h}, 72 \mathrm{~h}, 96 \mathrm{~h}, \\
120 \mathrm{~h} \text { post) }\end{array}$ & $\begin{array}{c}\text { CRP (pre, post-run } \leftrightarrow, 24 \mathrm{~h} \uparrow * \\
48 \mathrm{~h} \uparrow, 72 \mathrm{~h} \uparrow, 96 \mathrm{~h} \leftrightarrow 120) \text { Main } \\
\text { effect of time } \\
\text { VAS (pre, post-run }{ }^{*}, 24 \mathrm{~h} \uparrow * \\
48 \mathrm{~h} \uparrow *, 72 \mathrm{~h} \uparrow, 96 \mathrm{~h} \uparrow, 120 \mathrm{~h} \leftrightarrow \\
\text { pre-run) }\end{array}$ & $\begin{array}{l}\text { 6h IPC } \\
6 \mathrm{~h} \text { Con }\end{array}$ & $\begin{array}{l}\text { IPC }-90 \mathrm{mmHg} \text { for } \\
\text { cell } 1 \text { (distal) and } \\
\text { cell } 5 \text { (proximal) and } \\
100 \text { mmHg for cells } \\
2-4 \text { (compression } \\
30 \mathrm{~s} \text { ) }\end{array}$ \\
\hline $\begin{array}{l}\text { Northey et. } \\
\text { al / AU }\end{array}$ & $\begin{array}{l}\text { RCO / } \\
2016\end{array}$ & $\begin{array}{l}12 \text { strength- } \\
\text { trained male } \\
(24.0 \pm 6.3 \text { yrs })\end{array}$ & $\begin{array}{c}\mathrm{n}=12 \\
\\
\mathrm{n}=12 \\
\text { exp. } \\
\mathrm{n}=12 \\
\text { con. }\end{array}$ & $\begin{array}{l}\text { 1h post } \\
\text { exercises } \\
\text { SIPC } \\
\text { (45min) vs. } \\
\text { Placebo } \\
\text { therapy } \\
\text { (45 min) }\end{array}$ & $\begin{array}{c}10 \text { sets } \times 10 \text { rep. of } \\
\text { back squats at } 70 \% \\
1 \text { repetition } \\
\text { maximum }\end{array}$ & $\begin{array}{l}\text { f VAS, CON (peak } \\
\% \text { of quadriceps), } \\
\text { SJ, CMJ (Pre, } \\
\text { post, } 1 \mathrm{~h}, 24 \mathrm{~h})\end{array}$ & $\begin{array}{c}\text { CON peak (pre, post } \downarrow^{*}, 1 \mathrm{~h} \downarrow^{*}, \\
24 \mathrm{~h} \leftrightarrow) \\
\left.\text {, SJ (pre, post } \downarrow^{*}, 1 \mathrm{~h} \downarrow^{*}, 24 \mathrm{~h} \downarrow^{*}\right) \\
\left.\text { CMJ (pre, post } \downarrow^{*}, 1 \mathrm{~h} \downarrow^{*}, 24 \mathrm{~h} \downarrow\right) \\
\left.\text { VAS (pre, post } \uparrow^{*}, 1 \mathrm{~h} \uparrow^{*}, 24 \mathrm{~h} \uparrow^{*}\right)\end{array}$ & $\begin{array}{l}12 \mathrm{~min} \text { OCC - } \\
2 \text { sets } x \\
3 \mathrm{~min} \text { (per } \\
\text { leg) } \\
45 \mathrm{~min} \text { SIPC } \\
45 \mathrm{~min} \text { Con }\end{array}$ & $\begin{array}{r}\text { SIPC }-80 \mathrm{mmHg} \\
\text { (deflation - 15s) } \\
\text { OCC 220mmHg } \\
\text { (inflation 3min) }\end{array}$ \\
\hline $\begin{array}{l}\text { Heapy et. } \\
\text { al / NZ }\end{array}$ & $\begin{array}{l}\text { RCT / } \\
2018\end{array}$ & $\begin{array}{c}56 \\
\text { ultramarathon } \\
\text { ers (con. = 19; } e \\
42 \pm 9 \text { yrs),(IPC } \\
=18 ; 41 \pm 8 \\
\text { yrs), (Massage ( } \\
=19 ; 43 \pm 9 \\
\text { yrs), men }\end{array}$ & $\begin{array}{c}n=56 \\
\\
n=18 \\
\text { exp. (IPC) } \\
n=19 \\
\text { exp. } \\
\text { (Massage } \\
\text { ) } \\
n=19 \\
\text { con. }\end{array}$ & $\begin{array}{l}\text { Post-race, } \\
24 \mathrm{~h}, 48 \mathrm{~h}, \\
72 \mathrm{~h} \text { post- } \\
\text { race IPC } \\
\text { (20min) } \\
\text { ) post-race, } \\
24 \mathrm{~h}, 48 \mathrm{~h}, \\
72 \mathrm{~h} \text { post- } \\
\text { race } \\
\text { Massage } \\
\text { (25min) vs. } \\
\text { Placebo } \\
\text { therapy } \\
\text { (20 min) }\end{array}$ & $\begin{array}{l}\text { Run race - } 3 \\
\text { distance options of } \\
62.7 \mathrm{~km}, 87.4 \mathrm{~km} \text {, } \\
\text { and } 102.8 \mathrm{~km} \\
\end{array}$ & $\begin{array}{c}400 \mathrm{~m} \text { run times } \\
\text { (pre-race } 1 \text {, pre- } \\
\text { race } 2 \text {, post- } \\
\text { race at } 72 \mathrm{~h}, \\
\text { f } 120 \mathrm{~h}, 168 \mathrm{~h} \text {, and } \\
336 \mathrm{~h} \text { ), VAS, } \\
\text { Fatigue Scores } \\
\text { (pre, post, day } \\
24 \mathrm{~h}-168 \mathrm{~h} \text { post } \\
\text { and } 336 \mathrm{~h} \text { post) }\end{array}$ & $\begin{array}{c}400 \mathrm{~m} \text { run times (pre race 1, pre } \\
\text { race } 2 \leftrightarrow, 72 \mathrm{~h} \uparrow, 120 \mathrm{~h} \uparrow, \\
168 \mathrm{~h} \leftrightarrow, 336 \mathrm{~h} \leftrightarrow \text { ) Time effect* } \\
\text { (No group, or interaction effect) } \\
\text { VAS (pre race, post race } * *, \\
24 \mathrm{~h} \uparrow^{*}, 48 \mathrm{~h} \uparrow, 72 \mathrm{~h} \uparrow, 96 \mathrm{~h} \uparrow, \\
120 \mathrm{~h} \uparrow, 144 \mathrm{~h} \leftrightarrow, 168 \mathrm{~h} \leftrightarrow, \\
\text { d } \\
336 \mathrm{~h} \leftrightarrow \text { ) Time effect* (No group } \\
\text { or interaction effect) } \\
\text { Muscle Fatigue (pre-race, post- } \\
\text { race } *, 24 \mathrm{~h} \uparrow *, 48 \mathrm{~h} \uparrow *, \\
72 \mathrm{~h} \uparrow * \#, 96 \mathrm{~h} \uparrow \#, 120 \mathrm{~h} \uparrow \#, \\
144 \mathrm{~h} \leftrightarrow, 168 \mathrm{~h} \leftrightarrow, 336 \mathrm{~h} \leftrightarrow \text { ) Time } \\
\text { and interaction effect* (No group } \\
\text { effect) }\end{array}$ & $\begin{array}{l}80 \mathrm{~min} \text { IPC } \\
100 \mathrm{~min} \\
\text { Massage } \\
80 \mathrm{~min} \text { Con. }\end{array}$ & IPC - 80mmHg \\
\hline $\begin{array}{l}\text { Chleboun } \\
\text { et. al / USA }\end{array}$ & $\begin{array}{l}\text { RCT / } \\
1995\end{array}$ & $\begin{array}{l}22 \text { college } \\
\text { women } \\
\text { students }(21.7 \\
\pm 0.7 \mathrm{yrs})\end{array}$ & $\begin{array}{c}n=22 \\
n=22 \\
\text { exp. (IPC) } \\
n=10 \\
\text { con. } \\
\text { (passive } \\
\text { rest) }\end{array}$ & $\begin{array}{c}\text { Post- } \\
\text { exercise, } \\
24 \mathrm{~h}, 48 \mathrm{~h}, \\
72 \mathrm{~h}, 96 \mathrm{~h}, \\
120 \mathrm{~h} \text { post } \\
\text { IPC } \\
\text { (20min) vs. } \\
\text { Placebo } \\
\text { therapy } \\
\text { (20min) }\end{array}$ & $\begin{array}{c}3 \text { sets of ECC } \\
\text { exercise } \\
\text { performed with } \\
\text { weights equal to } \\
90 \%, 80 \% \text {, and } \\
70 \% \text { of the ISO } \\
\text { MVC }\end{array}$ & $\begin{array}{l}\text { Pain (five-point } \\
\text { pain rating } \\
\text { scale), Swelling } \\
\text { (post, day } 1 \text { to } \\
\text { 5), Stiffness, and } \\
\text { Isometric } \\
\text { Strength (pre- } \\
\text { exercise, pre, } \\
\text { post IPC days } 1 \\
\text { to } 5 \text { ) }\end{array}$ & 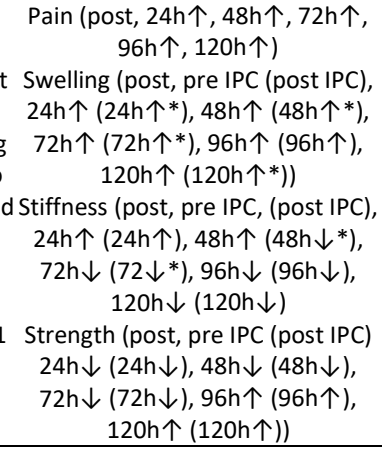 & $120 \mathrm{~min} I P C$ & $\begin{array}{l}\text { IPC - 60mmHg } \\
\text { (inflation 40s / } \\
\text { deflation 20s) }\end{array}$ \\
\hline $\begin{array}{l}\text { Velanzuela } \\
\text { et. al / ES }\end{array}$ & $\begin{array}{l}\text { RCO / } \\
2018\end{array}$ & $\begin{array}{l}10 \text { healthy } \\
\text { participants } \\
(27 \pm 4 \text { yrs), } 7\end{array}$ & $\begin{array}{l}n=10 \\
n=10 \\
\text { exp. }\end{array}$ & $\begin{array}{c}\text { post } \\
\text { exercises, } \\
24 \text { post- } \\
\text { EECP }\end{array}$ & $\begin{array}{c}\text { Plyometric } \\
\text { exercise bout (10 } \\
\text { sets of } 10 \text { jumps) } \\
\end{array}$ & $\begin{array}{c}\text { Muscle } \\
\text { Soreness (VAS), } \\
\text { CK, CMJ, RSI }\end{array}$ & $\begin{array}{c}\text { Muscle Soreness (pre, } 24 \mathrm{~h} \text { post } \uparrow, \\
48 \mathrm{~h} \text { post } \uparrow \text { ) CK (pre, } 24 \mathrm{~h} \text { post } \uparrow, \\
48 \mathrm{~h} \text { post } \uparrow \text { ) } \\
\text { CMJ (pre, } 24 \mathrm{~h} \text { post } \downarrow, 48 \mathrm{~h}\end{array}$ & $\begin{array}{l}\text { 60min EECP } \\
60 \mathrm{~min} \text { Con. }\end{array}$ & EECP $-80 \mathrm{mmHg}$ \\
\hline
\end{tabular}




\begin{tabular}{|c|c|c|c|c|c|c|c|c|c|}
\hline & & $\begin{array}{l}\text { men, } 3 \\
\text { females }\end{array}$ & $\begin{array}{l}\mathrm{n}=10 \\
\text { con. }\end{array}$ & $\begin{array}{l}\text { (30min) vs. } \\
\text { Placebo } \\
\text { therapy } \\
\text { (30 min) }\end{array}$ & & $\begin{array}{l}\text { (pre and } 24 \text { and } \\
48 \text { h post) }\end{array}$ & $\begin{array}{c}\text { post } \leftrightarrow \text { ) } \\
\text { RSI (pre, } 24 \mathrm{~h} \text { post } \downarrow, 48 \text { host } \leftrightarrow)\end{array}$ & & \\
\hline $\begin{array}{l}\text { Haun C.T. } \\
\text { et. al / USA }\end{array}$ & $\begin{array}{l}\mathrm{RCT} / \\
2017\end{array}$ & $\begin{array}{c}20 \text { resistance- } \\
\text { trained male } \\
(21.6 \pm 2.4 \text { yrs })\end{array}$ & $\begin{array}{c}n=10 \\
n=10 \\
\text { exp. } \\
(E P C) \\
n=10 \\
\text { con. }\end{array}$ & $\begin{array}{c}48 \mathrm{~h}, 72 \mathrm{~h}, \\
96 \mathrm{~h}, 120 \mathrm{~h}, \\
144 \mathrm{~h} \text { post } \\
\text { EPC (1h) } \\
\text { vs. Placebo } \\
\text { therapy } \\
\text { (1h) }\end{array}$ & $\begin{array}{l}10 \text { sets of } 5 \text { rep. at } \\
80 \% \text { of back squat } \\
1 \mathrm{RM}\end{array}$ & $\begin{array}{l}\text { CK, Flexibility } \\
\text { (pre, } 48 \mathrm{~h}-168 \mathrm{~h} \\
\text { post) CRP (pre, } \\
8 \mathrm{~h}-168 \mathrm{~h} \text { post) }\end{array}$ & 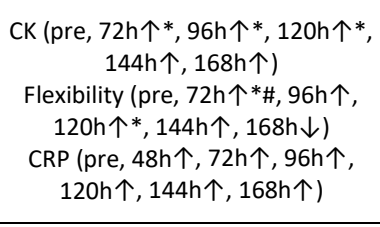 & $\begin{array}{l}\text { 5h EPC } \\
\text { 5h Con. }\end{array}$ & $\begin{array}{l}\mathrm{EPC} \sim 70 \mathrm{mmHg} \\
\text { (inflation - 30s / } \\
\text { deflation - 30s) }\end{array}$ \\
\hline $\begin{array}{l}\text { Oliver et. al } \\
\quad \text { / NZ }\end{array}$ & $\begin{array}{l}\mathrm{RCO} / \\
2021\end{array}$ & $\begin{array}{l}11 \text { well-trained } \\
\text { wheelchair } \\
\text { basketball and } \\
\text { rugby athletes } \\
(33 \pm 10 \text { yrs) } \\
\text { men }\end{array}$ & $\begin{array}{c}n=11 \\
n=11 \\
\text { exp. } \\
n=11 \\
\text { con. }\end{array}$ & $\begin{array}{c}\text { post } \\
\text { exercises } \\
\text { ISPC } \\
\text { (20min) vs. } \\
\text { Placebo } \\
\text { therapy } \\
\text { (30min) }\end{array}$ & $\begin{array}{l}10 \text { wheelchair } \\
\text { court sprints } \\
(28 \mathrm{~m}) .10 \text { times } \\
\text { figure of } 8 \text { agility } \\
\text { drill (the } 30 \mathrm{~s}) .10 \\
\text { sprints }(28 \mathrm{~m}) \\
\text { immediately } \\
\text { followed by } 3 \\
\text { medicine ball chest } \\
\text { throws }\end{array}$ & $\begin{array}{l}\text { Medicine Ball } \\
\text { Throw (m), } \\
\text { Wheelchair } \\
\text { Sprint, 5, 10, } 15 \\
\text { (m) (pre-ex, } \\
\text { post-ex, post- } \\
\text { rec) Muscle } \\
\text { Soreness 0-10 } \\
\text { scale and } \\
\text { Muscle Fatigue } \\
\text { 0-10 scale (pre- } \\
\text { ex, post-ex, } \\
\text { post-rec, 24h } \\
\text { post-rec) Blood } \\
\text { Lactate (post- } \\
\text { ex, post-rec) } \\
\end{array}$ & 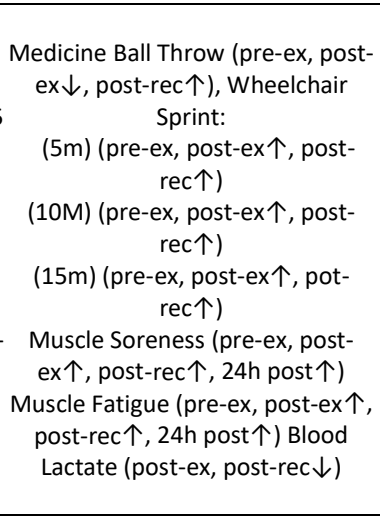 & $\begin{array}{l}\text { 20min ISPC } \\
\text { 30min Con. }\end{array}$ & $\begin{array}{r}\text { ISPC }-80 \mathrm{mmHg} \\
\text { (inflation 30s / } \\
\text { deflation 15s) }\end{array}$ \\
\hline $\begin{array}{l}\text { Cranston } \\
\text { et. al }\end{array}$ & $\begin{array}{l}\mathrm{RCT} / \\
2020\end{array}$ & $\begin{array}{c}50 \text { resistance- } \\
\text { trained } \\
\text { athletes ( } 27 \pm \\
4 \text { yrs), } 37 \text { men, } \\
13 \text { females }\end{array}$ & $\begin{array}{c}n=50 \\
n=25 \\
\text { exp. } \\
n=25 \\
\text { con. }\end{array}$ & $\begin{array}{c}\text { post } \\
\text { exercises } \\
\text { ISPC } \\
\text { (30min) vs. } \\
\text { Placebo } \\
\text { therapy } \\
\text { (30min) }\end{array}$ & $\begin{array}{c}\text { Fatiguing Exercise } \\
\text { Circuit (consisted } \\
\text { of } 5 \text { different } \\
\text { exercises): } \\
\text { 1. Reverse grip } \\
\text { battle rope waves } \\
\text { (the 60s) } \\
\text { 2. } 20 \text { m Farmers } \\
\text { carry (20 kg for } \\
\text { women and } 30 \text { kg } \\
\text { for men) } \\
\text { 3. Chin-ups } \\
\text { (maximum number } \\
\text { of repetitions) } \\
4 . \text { Chin-up bar } \\
\text { hangs (long as } \\
\text { possible with their } \\
\text { hands in a } \\
\text { pronated grip) } \\
\text { 5. Handgrip } \\
\text { crushers (as many } \\
\text { times as possible) }\end{array}$ & $\begin{array}{l}\text { Grip Strength } \\
\text { Dynamometer } \\
\text { (kg), Single-Arm } \\
\text { Medicine Ball } \\
\text { Throw (m), } \\
\text { Preacher Bench } \\
\text { rBicep Curls- max } \\
\text { repetitions (pre- } \\
\text { ex, post-ex, } \\
\text { post-rec) } \\
\end{array}$ & $\begin{array}{c}\text { Grip Strength Dynamometer (pre- } \\
\text { ex, post-ex } \downarrow \text {, post-rec } \downarrow \text { ) Single- } \\
\text { Arm Medicine Ball Throw (pre-ex, } \\
\text { post-ex } \downarrow \text {, post-rec } \uparrow \text { ) } \\
\text { Max. Rep. Single-Arm Preacher } \\
\text { Bench Bicep Curls (pre-ex, post- } \\
\text { ex } \downarrow \text {, post-rec } \downarrow \text { ) } \\
\text { Triceps Brachii Long Head } \\
\text { Soreness (pre-ex, post-ex } \uparrow, \text { post- } \\
\text { rec } \# \text { \#, 24h post-rec } \uparrow \# \text { ) } \\
\text { Biceps Brachii Soreness (pre-ex, } \\
\text { post-ex } \uparrow, \text { post-rec } \downarrow \#, \\
\text { post-rec } \uparrow \# \text { ) } \\
\text { Extensor Digitorum Soreness (pre- } \\
\text { ex, post-ex } \uparrow, \text { post-rec } \downarrow \#, 24 h \\
\text { post-rec } \uparrow \# \text { ) } \\
\text { Flexor Carpi Radialis Soreness } \\
\text { (pre-ex, post-ex } \uparrow, \text { post-rec } \downarrow \#, \\
24 h \text { post-rec } \uparrow \# \text { ) }\end{array}$ & $\begin{array}{l}30 \mathrm{~min} \text { ISPC } \\
\text { 30min Con. }\end{array}$ & $\begin{array}{l}\text { ISPC - 80mmHg } \\
\text { (inflation - 26s / } \\
\text { deflation - 15s) }\end{array}$ \\
\hline
\end{tabular}

Abbreviations: PCD (pneumatic compression device), CS ( compression sleeve), PC (pneumatic compression), EPC (external pneumatic compression), ECP (External counterpulsation), EECP (Enhanced external counterpulsation), IPC (intermittent pneumatic compression), ISPC (intermittent sequential pneumatic compression), OCC (evaluate vascular occlusion), SIPC (sequential intermittent pneumatic compression), VJ (vertical jump), SJ (squat jump), CK (creatine kinase), LDH (lactate dehydrogenase), ISO (isometric), CON (concentric), ECC (eccentric), HIIT (high intensity interval training), HIE (high-intensity exercise), CMJ (countermovement jump), DEC (deceleration), AMRAP (as much repetitions as possible), ALAP (as long as possible), WAnT (wingate anaerobic test), THB (total hemoglobin), O2HB (oxyhemoglobin), HHB (deoxyhemoglobin), ROM (range of motion), C (cortisol), T (testosterone), IgA (immunoglobulin-A), sAA (salivary alpha-amylase), CRP (C-reactive protein), PkP (peak power), AP (average power), FI (fatigue index), BLa (blood lactate concentration), NRS (numeric rating scale), CWI (cold water immersion), MuscleMechFx (muscle mechanical function), RPE (rate of perceived exertion), DM (Muscle radial deformation), TC (time of contraction), BF (biceps femoris), RF (rectus femoris), RSI (reactive strength index). \#-significant difference between groups, $\mathrm{p}<0.05 \uparrow^{*}$ - significant increase, $\mathrm{p}<0.05 \downarrow^{*}$ significant decrease, $\mathrm{p}<0.05 \biguplus$ - no significant change. 
Considerable variation was observed in therapy parameters among the studies. Intermittent sequential pneumatic compression (ISPC) was used in three studies [13][28][32]. Time of therapy was $2 \mathrm{~min}$. [3], 30s/15s [28] or 26s/15s [32]. External pneumatic compression (EPC) was used in three studies [12][30][31], two authors used the same parameters $70 \mathrm{mmHg}$ inflation - 30s, deflation -30s [12][30] and one study $235 \mathrm{mmHg}$ pressure [31]. The most popular therapy was IPC [29][18][25][19].

There was a different time of experimental and control condition, the majority did therapy post and after $24 \mathrm{~h}$. The average therapy session was $30 \mathrm{~min}$. The shortest time was 6 min. [26] and the maximum of $1 \mathrm{~h}$ [12][30][25]. Total therapeutic exposition time varied from 20 - $30 \mathrm{~min}$. [13][28][32] to longer time from $80 \mathrm{~min}$. to $6 \mathrm{~h}[29][18][19][29]$.

Outcome variables and time of measurement varied depending on the study. The period of measurement keeps on from Po-E [13][31][25][26][19][28][32] to 336h after exercise [29]. The average time of access outcomes was $48 \mathrm{~h}$. Muscle pain soreness and (CK) were the most often measured. Six studies investigated CK [12][30][18][31][27], five MS [12][13][27][28][32] and eight pain Visual analogue scale (VAS) [29][13][12][31][25][19][26][27]. Other authors access Over Fatigue [13], Flexibility [12], Muscle Dynamometry and vertical jump (VJ) [18][19] C-reactive protein (CRP) [25] [30], countermovement jump (CMJ), reactive strength index (RSI) [31][26][27], cortisol, testosterone, alpha-amylase and immunoglobulin [31]. Detailed information about the measured parameters can be observed in Table 1.

Main effects were measured Po-E through 336h after. CK increasing Po-E to 24h [31], 72h [18] and 168h [30]. Haun (2017) concluded that after $168 \mathrm{~h}$ there was no significant change. Significant effect was observed after 24h [18][31] and 96h [12][30] and 120h [30].

Muscle Pain increased Po-E to 24h [31][26], 96h [25], 120h [29][19] and 168h [13]. Significant effect was observed after one hour [26], 24h [29][31][26], 48h [25], 96h [13]. In one study a increasing was observed Po-E to $144 \mathrm{~h}$ but with no significant changes [19].

Muscle soreness had a heterogeneous direction of changes. Some authors observed decreasing after exercise from $72 \mathrm{~h}$ to $144 \mathrm{~h}$ and significant changes were measured after 72h and 120h [12] [29]. The majority observed significantly increasing MS Po-E and after $24 \mathrm{~h}$ to $96 \mathrm{~h}$ [13]. Velanzuela (2018) observed increasing MS after 24 and 48h but without any significant changes. Oliver (2021) observed increasing MS Po-E, post-recovery, and after $24 \mathrm{~h}$ and also without any significant changes. Cranston (2020) observed increasing Po-E in all four muscles group, post- recover decreasing in three groups with significant differences between groups, and after $24 \mathrm{~h}$ increasing in all four muscle groups, with significant differences between groups.

Hoffman (2016) observed that muscle fatigue increases postrace, posttreatment - significantly and reached significant difference between groups and postrace $24-168 \mathrm{~h}$. Two other authors analyzed change of these parameters [29][28] and Heapy (2018) observed changes post-race, $24-168 \mathrm{~h}$, and 336h after exercise and post-race, $24-72 \mathrm{~h}$ increase was significant, furthermore, $72 \mathrm{~h}, 96 \mathrm{~h}$ and $120 \mathrm{~h}$ was a significant difference between groups. In Oliver et.al (2021) muscle fatigue Po-E, post-recovery, and 24h Po-E remained unchanged.

Two studies assess muscle flexibility parameters [12][30]. Both observed increasing after $72 \mathrm{~h}$ and decreasing after $168 \mathrm{~h}$. Swelling and stiffness were observed by Chleboun et.al (1995) after 24-96h and 120h. The stiffness increased after 24 and $48 \mathrm{~h}$ and then decreased to $120 \mathrm{~h}$.

Two studies measured isometric strength [18][19]. Cochrane (2013) observed decreased peak isometric strength after $24 \mathrm{~h}$ and increased after 48 and $72 \mathrm{~h}$ - all changes were significant. Chleboun (1995) observed a decrease after 24- 72h and an increase after 96 and $120 \mathrm{~h}$.

Cochrane et.al (2013) measured a few dynamometry parameters: Peak concentric $30^{\circ}$ - decreased after 24,48 , and $72 \mathrm{~h}$; peak concentric $180^{\circ}$ decreased like previously parameters; peak ECC $30^{\circ}$ and $180^{\circ}$ - decreased after $24 \mathrm{~h}$ and increased after 48 and $72 \mathrm{~h}$. Other parameters average concentric $30^{\circ}, 180^{\circ}$ decreased after $24-72 \mathrm{~h}$; average ECC $30^{\circ}$, 
$180^{\circ}$ decreased after $24 \mathrm{~h}$ and increased after 48-72h. Northey et al. (2016) also measured concentric peak and he observed decreased post and after $1 \mathrm{~h}$ and then no significant changes.

Collins et al. (2019) assessed blood test results: cortisol, testosterone, immunoglobulin- were increased Po-E and decreased after 24h; Alpha-amylase - significant changes post and $24 \mathrm{~h}$ and between groups.. Oliver et al. (2021) measured blood lactate - post-recovery it decreased. C-reactive protein was measured in two studies [30][25] and remained unchanged after $24-144 \mathrm{~h}$ and $168 \mathrm{~h}$.

Some authors used exercises to measure the main effect. Hoffman et al. (2016) and Heapy et al. (2018) used $400 \mathrm{~m}$ runs with increased time after 72h [13] and 120h [29], and decreased time after 120h [13]. Another activity to measure effects was $6 \mathrm{~km}$ run after $168 \mathrm{~h}$ Po-E. In a countermovement jump (CM) [31][26][27] heterogenous results were observed: decreased post and increased after $24 \mathrm{~h}$ - significant changes between groups [31]. Decreased post, 1 and $24 \mathrm{~h}$-post and $1 \mathrm{~h}$ significant changes [26]. After $24 \mathrm{~h}$ decreased and $48 \mathrm{~h}$ no significant changes [27]. Valenzuela et al. (2018) also measured reactive strength index and had the same results as in the CMJ case. Cochrane et.al (2013) observed changes in vertical jump height - it decreased after $24 \mathrm{~h}$ and increased after $48 \mathrm{~h}$ and $72 \mathrm{~h}$; vertical jump peak power-decreased after 24-72h. Northey et al. (2016) used squat jump (SJ) to measure the main effect and noted only decreased post and after 1 and 24h. Oliver et al. (2021) used a medicine ball throw test and wheelchair sprint on 5, 10, 15 meters and observed decrease with post-recovery increase [28]. Sprint on every distance was increased. Cranston et al. (2020) used exercises: Grip strength dynamometer -decreased Po-E and post-recovery; Single-arm medicine ball throw - Po-E it decreased and then post-recovery increased; Max repetition single-arm preacher biceps curls - Po-E and recovery it decreased.

\subsection{Subgroup analysis}

Muscle soreness.

There was moderate and statistically significant reduction in MS in overall effect from 24-96h after DOMS induction in pressotherapy intervention (Standard Mean Difference $(\mathrm{SMD})=-0.33,95 \%$ CI $-0.49,-0.18 ; \mathrm{p}<0.0001 ; \mathrm{I}^{2}=7 \%$ ). In the Subgroup $24 \mathrm{~h}$ Po-E (participants $=311$; studies $=9)$ there was moderate but NS reduction in MS $(\mathrm{SMD}=-0.28,95 \%$ CI -0.60, 0.04; $\mathrm{p}=0.09 ; \mathrm{I}^{2}=43 \%$ ), 48h Po-E (participants $=144$; studies $=5$ ) there was moderate and significant reduction in MS (SMD $=-0.40,95 \% \mathrm{CI}-0.73,0.07 ; \mathrm{p}=0.02 ; \mathrm{I}^{2}=0 \%$ ), $72 \mathrm{~h}$ Po-E (participants $=124$; studies $=4$ ) there was moderate but NS reduction in MS $\left(\mathrm{SMD}=-0.37,95 \% \mathrm{CI}-0.79,0.05 ; \mathrm{p}=0.08 ; \mathrm{I}^{2}=24 \%\right)$ and $96 \mathrm{~h}$ Po-E (participants = 124; studies $=4$ ) there was moderate but NS reduction in MS. In overall effect from 24-96h heterogeneity was small $\left(\mathrm{I}^{2}=7 \% ; \chi^{2}=22.6, \mathrm{df}=21 ; \mathrm{p}=0.96\right)$. Only in the subgroup $24 \mathrm{~h}$ Po-E we detected NS heterogeneity $\left(\mathrm{I}^{2}=43 \% ; \chi^{2}=14.16, \mathrm{df}=8 ; \mathrm{p}=0.08\right) .48 \mathrm{~h}-96 \mathrm{~h}$ heterogeneity was low. Subgroup analysis from $24 \mathrm{~h}$ to $96 \mathrm{~h}$ didn't reveal statistically significant difference ( $p$ $=0.96$ ) (figure 4 ). 


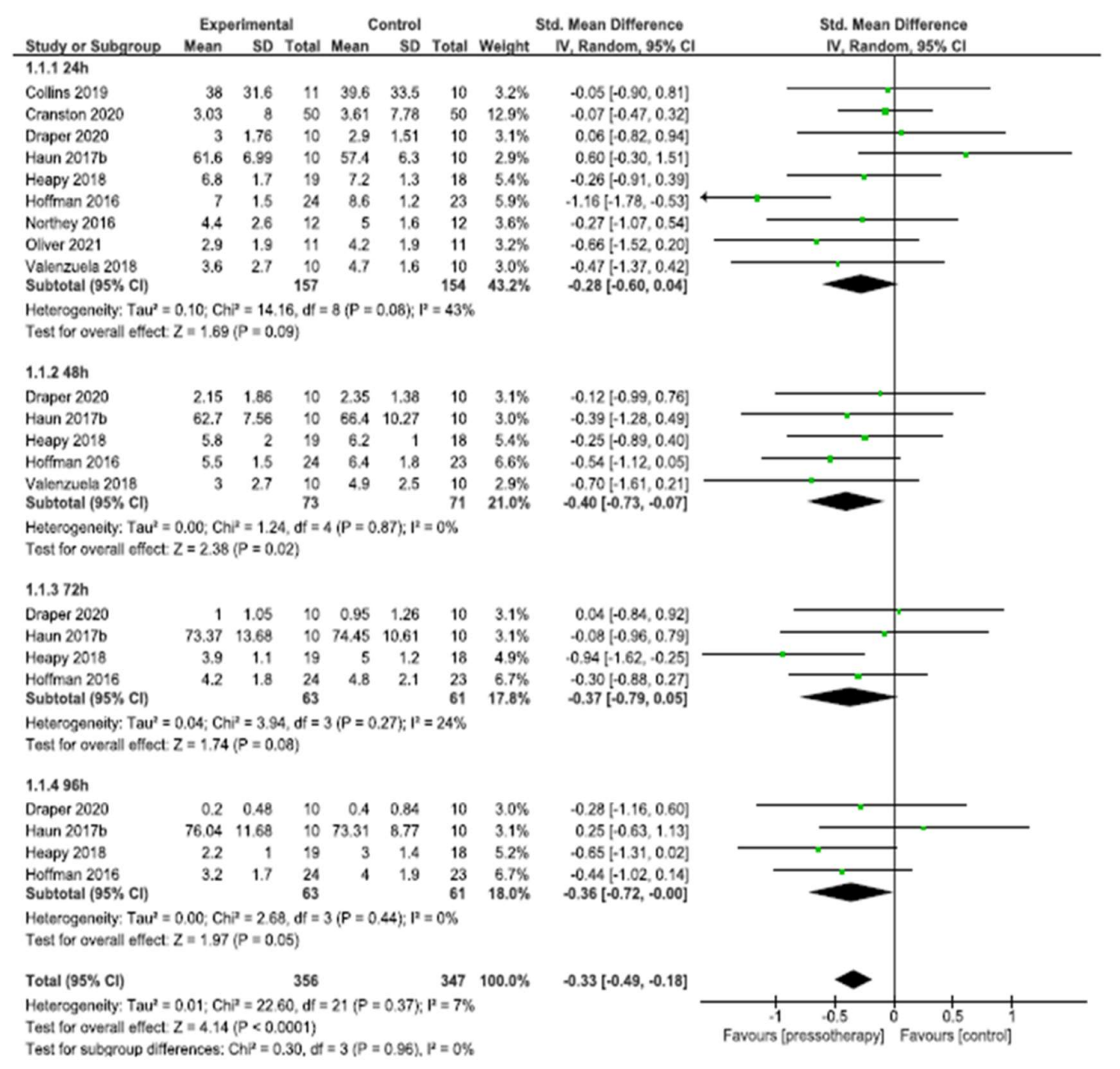

Figure 4. Effects of pressotherapy on muscle soreness from $24 \mathrm{~h}$ to $96 \mathrm{~h}$ after exercise.

Jump performance

24h Po-E (participants $=84 ;$ studies $=4 ; \mathrm{SMD}=-0.05,95 \% \mathrm{CI}-0.47,-0.38 ; \mathrm{p}=0.99 ; \mathrm{I}^{2}=$ $0 \%$ ), 48h Po-E (participants $=40 ;$ studies $=2 ; \mathrm{SMD}=-0.01,95 \% \mathrm{CI}-0.61,0.63 ; \mathrm{p}=0.77 ; \mathrm{I}^{2}=$ $0 \%$ ) and 72h Po-E (participants $=20$; studies $=1$; SMD $=-0.10,95 \%$ CI $-0.98,0.78 ; \mathrm{p}=0.82$; $\mathrm{I}^{2}=$ not applicable) there was a small statistically NS effect of pressotherapy on jump height. In overall effect from $24-72 \mathrm{~h}(\mathrm{SMD}=-0.04,95 \% \mathrm{CI}-0.36,-0.29 ; \mathrm{p}=0.82)$ heterogeneity was small $\left(\mathrm{I}^{2}=0 \% ; \chi^{2}=0.25, \mathrm{df}=21 ; \mathrm{p}=1.00\right)$. 
Subgroup analysis from $24 \mathrm{~h}$ to $96 \mathrm{~h}$ didn't reveal a statistically significant difference $(p=0.98)$.

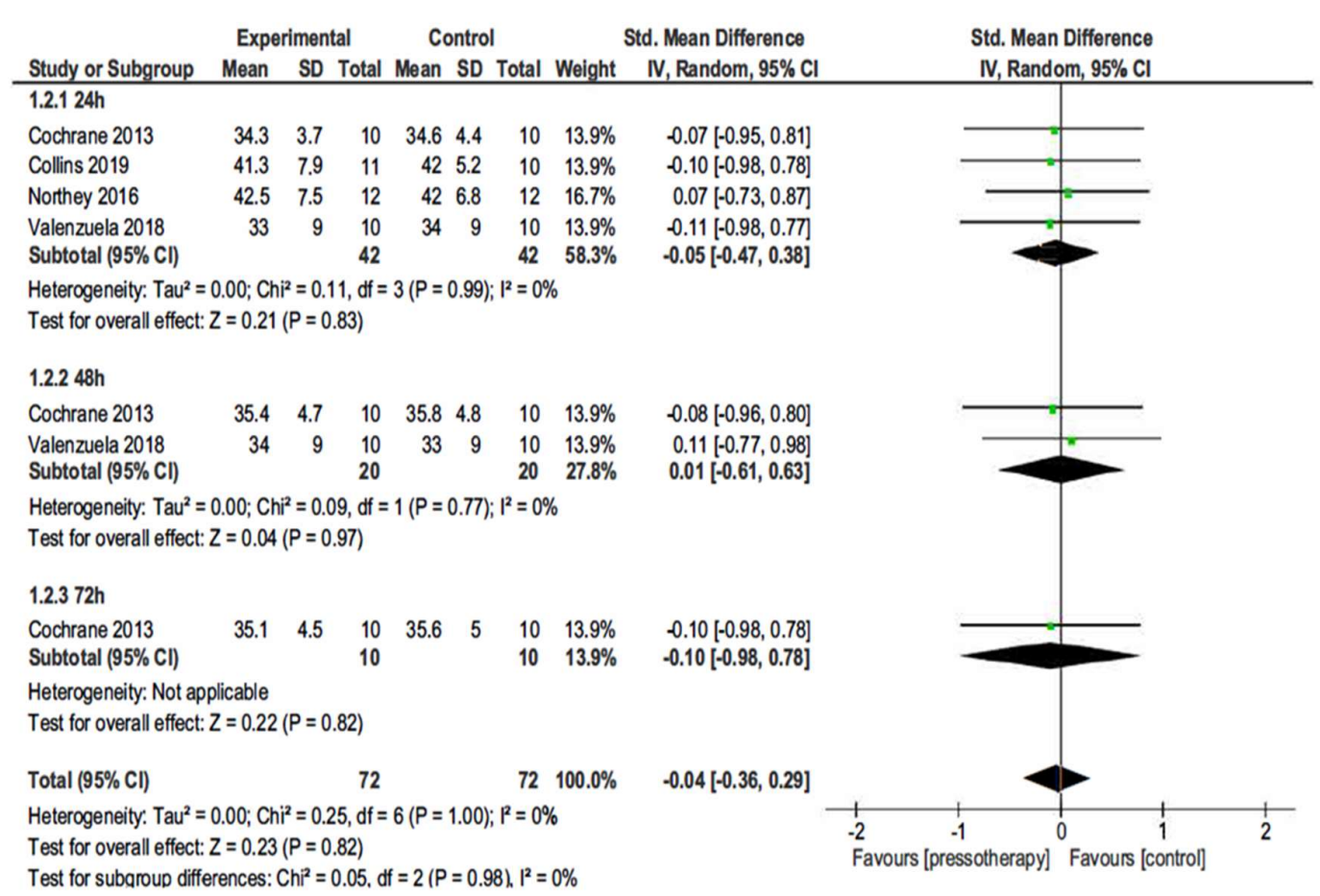

Figure 5. Effects of pressotherapy on jump performance from $24 \mathrm{~h}$ to $96 \mathrm{~h}$ after exercise. SMDs are calculated from CMJ, VJ, etc.

Creatine kinase

There was NS increase in serum CK activity in overall effect from 24-96h after DOMS induction in pressotherapy intervention (SMD $=0.41,95 \% \mathrm{CI}-0.07,0.89 ; \mathrm{p}=0.09 ; \mathrm{I}^{2}=63 \%$ ). In the subgroup 24h Po-E (participants $=81 ;$ studies $=4 ; \mathrm{SMD}=0.14,95 \% \mathrm{CI}-0.30,0.58 ; \mathrm{p}$ $\left.=0.54 ; \mathrm{I}^{2}=0 \%\right)$, 48h Po-E (participants $=60 ;$ studies $=3 ; \mathrm{SMD}=0.52,95 \% \mathrm{CI}-0.77,1.81 ; \mathrm{p}=$ $0.43 ; \mathrm{I}^{2}=82 \%$ ), 72h Po-E (participants $=40 ;$ studies $=2 ; \mathrm{SMD}=0.49,95 \% \mathrm{CI}-1.25,2.23 ; \mathrm{p}=$ $\left.0.58 ; \mathrm{I}^{2}=85 \%\right)$ there was small $(24 \mathrm{~h})$ and moderate $(48-72 \mathrm{~h})$ but NS increase in serum CK activity. 96h Po-E (participants = 20; studies $=1$ ) there was large and significant increase in CK activity for the pressotherapy group $\left(\mathrm{SMD}=1.26,95 \% \mathrm{CI} 0.28,2.23 ; \mathrm{p}=0.01\right.$; $\mathrm{I}^{2}=$ not applicable)

In overall effect from 24-96h heterogeneity was moderate $\left(\mathrm{I}^{2}=63 \% ; \chi^{2}=24.47, \mathrm{df}=9\right.$; $\mathrm{p}=0.004)$. Only in the subgroup $24 \mathrm{~h}$ Po-E we detected homogeneity $\left(\mathrm{I}^{2}=0 \% ; \chi^{2}=2.44\right.$, df $=3 ; \mathrm{p}=0.49) .48 \mathrm{~h}\left(\mathrm{I}^{2}=82 \% ; \chi^{2}=11.05, \mathrm{df}=2 ; \mathrm{p}=0.004\right)$ and $72 \mathrm{~h}\left(\mathrm{I}^{2}=85 \% ; \chi^{2}=6.78, \mathrm{df}=1\right.$; $\mathrm{p}=0.009$ ) heterogeneity was large. Subgroup analysis from $24 \mathrm{~h}$ to $96 \mathrm{~h}$ didn't reveal statistically significant difference $(p=0.23)$. 


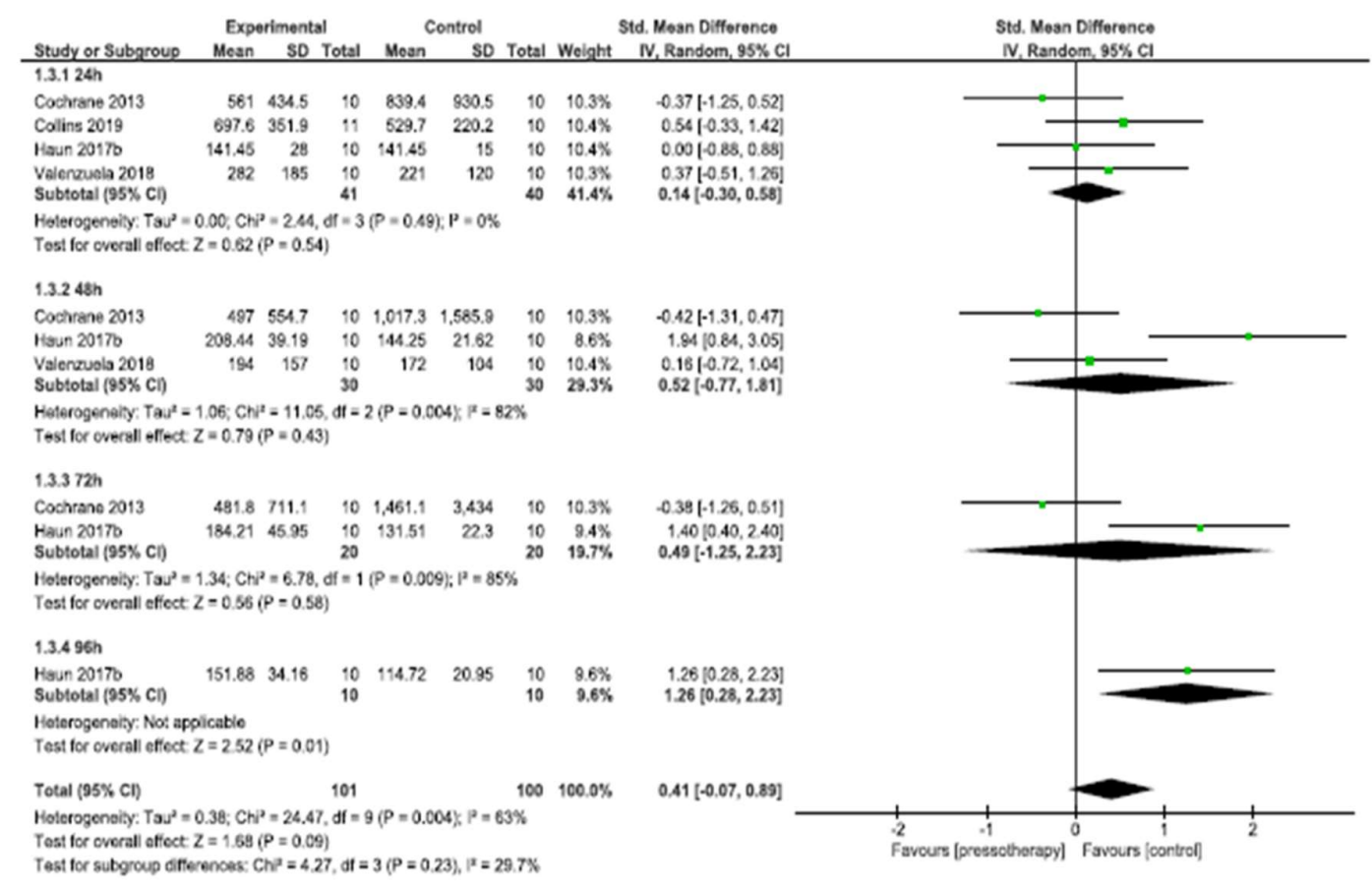

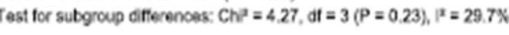

Figure 6. Effects of pressotherapy on serum CK activity from $24 \mathrm{~h}$ to $96 \mathrm{~h}$ after exercise.

\section{Discussion}

Most of the studies used a one-time protocol to assess the time of post-workout regeneration. The most reliable method would be to use it multiple times under different conditions to maximize results accuracy[33].

The best methods of post-workout recovery are sleep and a proper $\operatorname{diet}[34][35]$. Additional methods can only be supplementary. For the assessment of the credibility of the studies, we recommend that the information on whether pressotherapy was the primary method or an addition to the more comprehensive scheme should be included in the methodology section.

Maximizing the efficiency of post-workout adaptation is crucial for athletes to maintain an appropriate performance level throughout the season and during the pre-competition preparation periods [36][37]. This is especially important in sports with a high frequency of competitions (i.e. team sports like soccer and basketball), as well as in disciplines where the athlete prepares for a long time for one event in which their organism achieves peak performance (i.e. individual disciplines such as sprinting or swimming).

We stipulate that pressotherapy does NS affect post-workout regeneration and can only supplement a complex protocol.

\section{Serum CK level}

The blood level of CK is an indicator of the status of muscle damage and change in both pathological and normal conditions [38]. An increase in this enzyme may predict a state of microscopic tissue impairment after acute and prolonged injuries. Variables in CK level are also observed under physiological conditions in athletes after demanding training. The highest $\mathrm{CK}$ growth is observed after prolonged exercise, i.e. triathlon events and demanding strength exercises, or activities that include eccentric muscle contraction phase, i.e downhill running [39][40]. In our study, we saw an improvement in this 
parameter, which suggests that pressotherapy improves regeneration. However, its impact was not statistically significant in any case except the 96h Po-E group, which had the lowest number of participants. In addition, a significant result was observed in the longest period after the training was performed, which leaves some ambiguity as CK activity decreases with time and it is a natural process [41]. Not without relevance is also the fact that a significant result was observed by Haun et. al., who investigated CK levels on a group of trained high-volume endurance athletes, who underwent over $70 \mathrm{~h}$ of exertion per week for 3 months. Although significant results have been observed, previous studies suggest that CK levels naturally decline between days 4 and 10 after exercise [42]. The characteristics of the test group (endurance athletes) and testing protocol could also affect the results, as resting CK levels are higher in the trained population [43][44] and everyday strenuous workouts may cause persistent blood rise of CK [45]. Therefore, the potential outcome of pressotherapy on a different group of people would not be so important. To summarize, in the current state of knowledge, pressotherapy should not be recommended as the basic method of recovery after exercise, because there is a large heterogeneity of previous research results.

\section{DOMS}

DOMS is a regular experience for advanced or beginner athletes. Its manifestations can range from muscle stiffness to severe excruciating pain [46]. DOMS is most prevalent at the beginning of the sporting season when athletes are returning to training following a period of reduced activity[47]. DOMS is also common when athletes are first introduced to certain types of activities regardless of the time of year. DOMS can negatively attenuate athletic performance [48]. Possible mechanisms include a reduction in joint ROM, peak torque, and feeling of pain [46]. Compensation methods may raise the probability of further injury [49][50] when participants try to return to activity too early without completing the full recovery process. Therefore, it is of high importance to search for new methods of the most effective regeneration and reduction of MS. Commonly described in the literature are pressotherapy, [46] stretching [51], cryotherapy [52], and massage, mainly considered as self-foam rolling. It has been the most often assessed parameter in selected studies. Although pressotherapy is one of the methods of DOMS reduction, our results indicate that its use for this purpose remains questionable. Only when MS was measured after $48 \mathrm{~h}$ a significant effect of pressotherapy was observed. This method does also significantly alleviates DOMS when considering the whole population and all protocols. On the other hand, no significant reduction in MS was found in the remaining groups. Taking into account the previously mentioned methods of therapy, which are easily available (stretching or foam-rolling), as well as low-cost (cryotherapy and water immersion) or self-applicable and physiologic (i.e. rest), there are few arguments in favor of the wide use of pressotherapy in the current state of knowledge. High prices and limited availability suggest other forms as a method of choice and first-line treatment strategy. However, pressotherapy has shown some positive effects, mainly limited to the $48 \mathrm{~h}$ Po-E period, so while the above-mentioned factors are not a barrier, it can be used in some circumstances [53] (e.g. in professional athletes as a supplemental method).

\section{Jump performance}

The level of muscle power in the lower limbs is a vital factor in numerous disciplines, such as sprinting [54][55] or in decisive moments of team sports [56][57]. In a widespread view, the research has demonstrated that jump heigh is an applicable index to characterize power output, mainly described by the association found between them [58]. It is meaningful that upright jump may be easily evaluated and hereafter used by team staff and physical trainers to categorize the level of athletes' muscle power within a wider group of participants.[59][60] Due to the great practical importance of jump performance in the overall assessment of an athlete's fitness and the development of motor skills, it is crucial 
to properly place this type of activity in the training plan and the microcycle.[61][62] Effective recovery after jumping efforts would be of key importance, hence the influence of pressotherapy on jump performance was also assessed in this meta-analysis. In our review, we did not observe any significant effect of pressotherapy on jump ability performed at various intervals from the previous exercise. Further investigation is needed to specify whether and in what population this method will be an effective approach for improving jump performance and overall power generation.

\section{Limitations}

Although, this paper has a few limitations. Firstly, we performed a comprehensive literature investigation whereas did exclude articles that were not published in English. However, from an actual point of view, we suppose this will have a minor effect on our outcomes.[63] Nevertheless, we conducted a reasonable attitude to overwhelm these barriers and attempted to stick to principles of open science. Secondly, the protocols used and the study groups differed between the selected articles. Third, the time of outcome evaluation from the preliminary endpoint was not identical in all trials. Fourth, the particular subgroup analyses were conceivably underpowered due to their small participant number and should be interpreted carefully. To enhance the validity of results in similar research, future randomized studies have to concentrate on better conducting and reporting of applied protocol and methodology, intention-to-treat examination, assessor blinding, random sequence generation, control group observation, and reporting of adverse events or the possible other influencing factors.

\section{Conclusions}

The conducted systematic review and meta-analysis assessed 12 randomized controlled studies investigating the outcome of pressotherapy on the recovery of absolute (i.e. physiological), and subjective (i.e. perceptual) outcomes. The findings indicate only moderate benefits of using pressotherapy as a recovery intervention, dependent on the type of exercise and used protocol. A reduction in DOMS, changes in CK level, and improvements in perceived recovery were observed after pressotherapy, although they were usually not significant. Dose-response relationships emerged for several variables indicating that different duration protocols may improve the efficacy of pressotherapy if applied after exercise. We recommend further continuing research on various populations and broadening tested protocols to obtain the highest possible homogeneity of results and to facilitate the creation of a consensus statement on whether pressotherapy seems to be an effective method in minimizing exercise-induced negative effects.

Author Contributions: Conceptualization, S.W. and M.C.; methodology, S.W., P.W.; writing-original draft preparation, P.W., S.W., P.K., M.C. and W.P.; software and statistics, S.W., writing-review and editing, M.J., M.C., B.M., P.K. and W.P.; supervision, S.W. and P.K. All authors have read and agreed to the published version of the manuscript.

Funding: This research received no external funding.

Data Availability Statement: Data are available from the corresponding author upon reasonable request.

Conflicts of Interest: The authors declare there are no conflict of interest.

\section{References}

1. Moher, D.; Liberati, A.; Tetzlaff, J.; Altman, D.G. Preferred Reporting Items for Systematic Reviews and MetaAnalyses: The PRISMA Statement. PLoS Med. 2009, 6, e1000097, doi:10.1371/journal.pmed.1000097.

2. Howatson, G.; van Someren, K.A. The Prevention and Treatment of Exercise-Induced Muscle Damage. Sport. Med. 2008, 38, 483-503, doi:10.2165/00007256-200838060-00004.

3. McAnulty, S.; McAnulty, L.; Nieman, D.; Morrow, J.; Dumke, C.; Henson, D. Effect of NSAID on Muscle Injury and Oxidative Stress. Int. J. Sports Med. 2007, 28, 909-915, doi:10.1055/s-2007-964966. 
4. Kim, J.; Lee, J. A review of nutritional intervention on delayed onset muscle soreness. Part I. J. Exerc. Rehabil. 2014, 10, 349-356, doi:10.12965/jer.140179.

5. Monedero, J.; Donne Effect of Recovery Interventions on Lactate Removal and Subsequent Performance. Int. J. Sports Med. 2000, 21, 593-597, doi:10.1055/s-2000-8488.

6. Tanner, R.K.; Fuller, K.L.; Ross, M.L.R. Evaluation of three portable blood lactate analysers: Lactate Pro, Lactate Scout and Lactate Plus. Eur. J. Appl. Physiol. 2010, 109, 551-559, doi:10.1007/s00421-010-1379-9.

7. Mair, J.; Mayr, M.; Mullet, E.; Koller, A.; Haid, C.; Artner-Dworzak, E.; Calzolari, C.; Larue, C.; Puschendorf, B. Rapid Adaptation to Eccentric Exercise-Induced Muscle Damage. Int. J. Sports Med. 1995, 16, 352-356, doi:10.1055/s-2007-973019.

8. Lieber, R.L.; Fridén, J. Morphologic and Mechanical Basis of Delayed-Onset Muscle Soreness. J. Am. Acad. Orthop. Surg. 2002, 10, 67-73, doi:10.5435/00124635-200201000-00009.

9. Gaitanos, G.C.; Williams, C.; Boobis, L.H.; Brooks, S. Human muscle metabolism during intermittent maximal exercise. J. Appl. Physiol. 1993, 75, 712-719, doi:10.1152/jappl.1993.75.2.712.

10. Machado, A.F.; Ferreira, P.H.; Micheletti, J.K.; de Almeida, A.C.; Lemes, Í.R.; Vanderlei, F.M.; Netto Junior, J.; Pastre, C.M. Can Water Temperature and Immersion Time Influence the Effect of Cold Water Immersion on Muscle Soreness? A Systematic Review and Meta-Analysis. Sport. Med. 2016, 46, 503-514, doi:10.1007/s40279-015-04317.

11. Cochrane, D.J. Effectiveness of using wearable vibration therapy to alleviate muscle soreness. Eur. J. Appl. Physiol. 2017, 117, 501-509, doi:10.1007/s00421-017-3551-y.

12. Haun, C.T.; Roberts, M.D.; Romero, M.A.; Osburn, S.C.; Mobley, C.B.; Anderson, R.G.; Goodlett, M.D.; Pascoe, D.D.; Martin, J.S. Does external pneumatic compression treatment between bouts of overreaching resistance training sessions exert differential effects on molecular signaling and performance-related variables compared to passive recovery? An exploratory study. PLoS One 2017, 12, e0180429, doi:10.1371/journal.pone.0180429.

13. Hoffman, M.D.; Badowski, N.; Chin, J.; Stuempfle, K.J. A Randomized Controlled Trial of Massage and Pneumatic Compression for Ultramarathon Recovery. J. Orthop. Sport. Phys. Ther. 2016, 46, 320-326, doi:10.2519/jospt.2016.6455.

14. Sands, W.A.; Murray, M.B.; Murray, S.R.; McNeal, J.R.; Mizuguchi, S.; Sato, K.; Stone, M.H. Peristaltic Pulse Dynamic Compression of the Lower Extremity Enhances Flexibility. J. Strength Cond. Res. 2014, 28, 1058-1064, doi:10.1519/JSC.0000000000000244.

15. Sands, W.A.; McNeal, J.R.; Murray, S.R.; Stone, M.H. Dynamic Compression Enhances Pressure-to-Pain Threshold in Elite Athlete Recovery. J. Strength Cond. Res. 2015, 29, 1263-1272, doi:10.1519/JSC.0000000000000412.

16. Muluk, S.C.; Hirsch, A.T.; Taffe, E.C. Pneumatic Compression Device Treatment of Lower Extremity Lymphedema Elicits Improved Limb Volume and Patient-reported Outcomes. Eur. J. Vasc. Endovasc. Surg. 2013, 46, 480-487, doi:10.1016/j.ejvs.2013.07.012.

17. Martin, J.S.; Friedenreich, Z.D.; Borges, A.R.; Roberts, M.D. Acute Effects of Peristaltic Pneumatic Compression on Repeated Anaerobic Exercise Performance and Blood Lactate Clearance. J. Strength Cond. Res. 2015, 29, 29002906, doi:10.1519/JSC.0000000000000928.

18. Cochrane, D.; Booker, H.; Mundel, T.; Barnes, M. Does Intermittent Pneumatic Leg Compression Enhance Muscle Recovery after Strenuous Eccentric Exercise? Int. J. Sports Med. 2013, 34, 969-974, doi:10.1055/s-0033-1337944.

19. Chleboun, G.S.; Howell, J.N.; Baker, H.L.; Ballard, T.N.; Graham, J.L.; Hallman, H.L.; Perkins, L.E.; Schauss, J.H.; Conatser, R.R. Intermittent pneumatic compression effect on eccentric exercise-induced swelling, stiffness, and strength loss. Arch. Phys. Med. Rehabil. 1995, 76, 744-749, doi:10.1016/S0003-9993(95)80529-X.

20. Zuj, K.A.; Prince, C.N.; Hughson, R.L.; Peterson, S.D. Enhanced muscle blood flow with intermittent pneumatic compression of the lower leg during plantar flexion exercise and recovery. J. Appl. Physiol. 2018, 124, 302-311, doi:10.1152/japplphysiol.00784.2017.

21. Kevin Stetter, E.H. An Intermittent Pneumatic Compression Device Reduces Blood Lactate Concentrations More Effectively Than Passive Recovery after Wingate Testing. J. Athl. Enhanc. 2013, 02, doi:10.4172/2324-9080.1000115.

22. Waller, T.; Caine, M.; Morris, R. Intermittent Pneumatic Compression Technology for Sports Recovery. In The Engineering of Sport 6; Springer New York: New York, NY, 2006; pp. 391-396.

23. Brown, D. A Review of the PubMed PICO Tool: Using Evidence-Based Practice in Health Education. Health Promot. Pract. 2020, 21, 496-498, doi:10.1177/1524839919893361.

24. Sterne, J.A.C.; Savović, J.; Page, M.J.; Elbers, R.G.; Blencowe, N.S.; Boutron, I.; Cates, C.J.; Cheng, H.-Y.; Corbett, M.S.; Eldridge, S.M.; et al. RoB 2: a revised tool for assessing risk of bias in randomised trials. BMJ 2019, 14898, doi:10.1136/bmj.14898. 
25. Draper, S.N.; Kullman, E.L.; Sparks, K.E.; Little, K.; Thoman, J. Effects of Intermittent Pneumatic Compression on Delayed Onset Muscle Soreness (DOMS) in Long Distance Runners. Int. J. Exerc. Sci. 2020, 13, 75-86.

26. Northey, J.M.; Rattray, B.; Argus, C.K.; Etxebarria, N.; Driller, M.W. Vascular Occlusion and Sequential Compression for Recovery After Resistance Exercise. J. Strength Cond. Res. 2016, 30, 533-539, doi:10.1519/JSC.0000000000001080.

27. Valenzuela, P.L.; Montalvo, Z.; Torrontegi, E.; Sánchez-Martínez, G.; Lucia, A.; de la Villa, P. Enhanced External Counterpulsation and Recovery From a Plyometric Exercise Bout. Clin. J. Sport Med. 2018, Publish Ah, doi:10.1097/JSM.0000000000000620.

28. Oliver, A.; Driller, M. The Use of Upper-Body Intermittent Sequential Pneumatic Compression Arm Sleeves on Recovery From Exercise in Wheelchair Athletes. Am. J. Phys. Med. Rehabil. 2021, 100, 65-71, doi:10.1097/PHM.0000000000001521.

29. Heapy, A.M.; Hoffman, M.D.; Verhagen, H.H.; Thompson, S.W.; Dhamija, P.; Sandford, F.J.; Cooper, M.C. A randomized controlled trial of manual therapy and pneumatic compression for recovery from prolonged running an extended study. Res. Sport. Med. 2018, 26, 354-364, doi:10.1080/15438627.2018.1447469.

30. Haun, C.T.; Roberts, M.D.; Romero, M.A.; Osburn, S.C.; Healy, J.C.; Moore, A.N.; Mobley, C.B.; Roberson, P.A.; Kephart, W.C.; Mumford, P.W.; et al. Concomitant external pneumatic compression treatment with consecutive days of high intensity interval training reduces markers of proteolysis. Eur. J. Appl. Physiol. 2017, 117, 2587-2600, doi:10.1007/s00421-017-3746-2.

31. Collins, R.; McGrath, D.; Horner, K.; Eusebi, S.; Ditroilo, M. Effect of External Counterpulsation on Exercise Recovery in Team Sport Athletes. Int. J. Sports Med. 2019, 40, 511-518, doi:10.1055/a-0923-9144.

32. Cranston, A.W.; Driller, M.W. Investigating the Use of an Intermittent Sequential Pneumatic Compression Arm Sleeve for Recovery After Upper-Body Exercise. J. Strength Cond. Res. 2020, Publish Ah, doi:10.1519/JSC.0000000000003680.

33. Wiecha, S.; Jarocka, M.; Wiśniowski, P.; Cieśliński, M.; Price, S.; Makaruk, B.; Kotowska, J.; Drabarek, D.; Cieśliński, I.; Sacewicz, T. The efficacy of intermittent pneumatic compression and negative pressure therapy on muscle function, soreness and serum indices of muscle damage: a randomized controlled trial. BMC Sports Sci. Med. Rehabil. 2021, 13, 144, doi:10.1186/s13102-021-00373-2.

34. Halson, S.L. Nutrition, sleep and recovery. Eur. J. Sport Sci. 2008, 8, 119-126, doi:10.1080/17461390801954794.

35. Kerksick, C.M.; Arent, S.; Schoenfeld, B.J.; Stout, J.R.; Campbell, B.; Wilborn, C.D.; Taylor, L.; Kalman, D.; SmithRyan, A.E.; Kreider, R.B.; et al. International society of sports nutrition position stand: nutrient timing. J. Int. Soc. Sports Nutr. 2017, 14, 33, doi:10.1186/s12970-017-0189-4.

36. Davis, J.K.; Oikawa, S.Y.; Halson, S.; Stephens, J.; O’Riordan, S.; Luhrs, K.; Sopena, B.; Baker, L.B. In-Season Nutrition Strategies and Recovery Modalities to Enhance Recovery for Basketball Players: A Narrative Review. Sport. Med. 2021, doi:10.1007/s40279-021-01606-7.

37. Kovacs, M.S.; Baker, L.B. Recovery interventions and strategies for improved tennis performance. Br. J. Sports Med. 2014, 48, i18-i21, doi:10.1136/bjsports-2013-093223.

38. Brancaccio, P.; Maffulli, N.; Limongelli, F.M. Creatine kinase monitoring in sport medicine. Br. Med. Bull. 2007, 81-82, 209-230, doi:10.1093/bmb/ldm014.

39. Noakes, T.D. Effect of Exercise on Serum Enzyme Activities in Humans. Sport. Med. 1987, 4, 245-267, doi:10.2165/00007256-198704040-00003.

40. Malm, C.; Sjödin, B.; Sjöberg, B.; Lenkei, R.; Renström, P.; Lundberg, I.E.; Ekblom, B. Leukocytes, cytokines, growth factors and hormones in human skeletal muscle and blood after uphill or downhill running. J. Physiol. 2004, 556, 983-1000, doi:10.1113/jphysiol.2003.056598.

41. Baird, M.F.; Graham, S.M.; Baker, J.S.; Bickerstaff, G.F. Creatine-Kinase- and Exercise-Related Muscle Damage Implications for Muscle Performance and Recovery. J. Nutr. Metab. 2012, 2012, 1-13, doi:10.1155/2012/960363.

42. Ehlers, G.G.; Ball, T.E.; Liston, L. Creatine Kinase Levels are Elevated During 2-A-Day Practices in Collegiate Football Players. J. Athl. Train. 2002, 37, 151-156.

43. Fallon, K.E.; Sivyer, G.; Sivyer, K.; Dare, A. The biochemistry of runners in a $1600 \mathrm{~km}$ ultramarathon. Br. J. Sports Med. 1999, 33, 264-269, doi:10.1136/bjsm.33.4.264.

44. Hortobágyi, T.; Denahan, T. Variability in Creatine Kinase: Methodological, Exercise, and Clinically Related Factors. Int. J. Sports Med. 1989, 10, 69-80, doi:10.1055/s-2007-1024878.

45. Kratz, A.; Lewandrowski, K.B.; Siegel, A.J.; Chun, K.Y.; Flood, J.G.; Van Cott, E.M.; Lee-Lewandrowski, E. Effect of Marathon Running on Hematologic and Biochemical Laboratory Parameters, Including Cardiac Markers. Am. J. Clin. Pathol. 2002, 118, 856-863, doi:10.1309/14TY-2TDJ-1X0Y-1V6V. 
46. Cheung, K.; Hume, P.A.; Maxwell, L. Delayed Onset Muscle Soreness. Sport. Med. 2003, 33, 145-164, doi:10.2165/00007256-200333020-00005.

47. Armstrong, R.B. Mechanisms of exercise-induced delayed onset muscular soreness: a brief review. Med. Sci. Sports Exerc. 1984, 16, 529-38.

48. Hamill, J.; Freedson, P.S.; Clarkson, P.M.; Braun, B. Muscle Soreness during Running: Biomechanical and Physiological Considerations. Int. J. Sport Biomech. 1991, 7, 125-137, doi:10.1123/ijsb.7.2.125.

49. Orchard, J.; Marsden, J.; Lord, S.; Garlick, D. Preseason Hamstring Muscle Weakness Associated with Hamstring Muscle Injury in Australian Footballers. Am. J. Sports Med. 1997, 25, 81-85, doi:10.1177/036354659702500116.

50. EDGERTON, V.R.; WOLF, S.L.; LEVENDOWSKI, D.J.; ROY, R.R. Theoretical basis for patterning EMG amplitudes to assess muscle dysfunction. Med. Eamp Sci. Sport. Eamp Exerc. 1996, 28, 744-751, doi:10.1097/00005768199606000-00013.

51. Wessel Effect of Stretching on the Intensity of Delayed-Onset Muscle Soreness. Clin. J. Sport Med. $1994,4,83-87$.

52. Gulick, D.T.; Kimura, I.F. Delayed Onset Muscle Soreness: What Is It and How Do We Treat It? J. Sport Rehabil. 1996, 5, 234-243, doi:10.1123/jsr.5.3.234.

53. Vairo, G.L.; Miller, S.J.; Rier, N.M.C.I.; Uckley, W.B.I. Systematic Review of Efficacy for Manual Lymphatic Drainage Techniques in Sports Medicine and Rehabilitation: An Evidence-Based Practice Approach. J. Man. Manip. Ther. 2009, 17, 80E-89E, doi:10.1179/jmt.2009.17.3.80E.

54. Harrison, A.J.; Keane, S.P.; Coglan, J. Force-Velocity Relationship and Stretch-Shortening Cycle Function in Sprint and Endurance Athletes. J. Strength Cond. Res. 2004, 18, 473, doi:10.1519/13163.1.

55. Loturco, I.; Pereira, L.A.; Cal Abad, C.C.; D'Angelo, R.A.; Fernandes, V.; Kitamura, K.; Kobal, R.; Nakamura, F.Y. Vertical and Horizontal Jump Tests Are Strongly Associated With Competitive Performance in 100-m Dash Events. J. Strength Cond. Res. 2015, 29, 1966-1971, doi:10.1519/JSC.0000000000000849.

56. Sattler, T.; Hadžić, V.; Dervišević, E.; Markovic, G. Vertical Jump Performance of Professional Male and Female Volleyball Players. J. Strength Cond. Res. 2015, 29, 1486-1493, doi:10.1519/JSC.0000000000000781.

57. Castagna, C.; Castellini, E. Vertical Jump Performance in Italian Male and Female National Team Soccer Players. J. Strength Cond. Res. 2013, 27, 1156-1161, doi:10.1519/JSC.0b013e3182610999.

58. Kons, R.L.; Ache-Dias, J.; Detanico, D.; Barth, J.; Dal Pupo, J. Is Vertical Jump Height an Indicator of Athletes' Power Output in Different Sport Modalities? J. Strength Cond. Res. 2018, 32, 708-715, doi:10.1519/JSC.0000000000001817.

59. Canavan, P.K.; Vescovi, J.D. Evaluation of Power Prediction Equations: Peak Vertical Jumping Power in Women. Medicine and Science in Sports and Exercise 2004, 36, 1589-1593, doi:10.1249/01.MSS.0000139802.96395.AC.

60. Potteiger, J.A.; Lockwood, R.H.; Haub, M.D.; Dolezal, B.A.; Almuzaini, K.S.; Schroeder, J.M.; Zebas, C.J.; Lockwood, R.; Dolezal, B.; Almuzaini, K.; et al. Muscle Power and Fiber Characteristics Following 8 Weeks of Plyometric Training. Journal of Strength and Conditioning Research 1999, 13, 275-279.

61. Bobbert, M.F. Drop Jumping as a Training Method for Jumping Ability. Sports medicine (Auckland, N.Z.) 1990, 9, 7-22, doi:10.2165/00007256-199009010-00002.

62. Markovic, G.; Markovic, G. Does Plyometric Training Improve Vertical Jump Height? A Meta-Analytical Review. British Journal of Sports Medicine 2007, 41, 349-355, doi:10.1136/BJSM.2007.035113.

63. Morrison, A.; Polisena, J.; Husereau, D.; Moulton, K.; Clark, M.; Fiander, M.; Mierzwinski-Urban, M.; Clifford, T.; Hutton, B.; Rabb, D. The Effect of English-Language Restriction on Systematic Review-Based Meta-Analyses: A Systematic Review of Empirical Studies. International journal of technology assessment in health care 2012, 28, 138144, doi:10.1017/S0266462312000086. 